\title{
Lagrangian-Hamiltonian unified formalism for field theory
}

\author{
Arturo Echeverría-Enríquez \\ Departamento de Matemática Aplicada IV, Edificio C-3, Campus Norte UPC, \\ C/Jordi Girona 1, E-08034 Barcelona, Spain \\ Carlos López a) \\ Departamento de Matemáticas, Campus Universitario. Fac. Ciencias, \\ 28871 Alcalá de Henares, Spain \\ Jesús Marín-Solano ${ }^{\text {b) }}$ \\ Departamento de Matemática Económica, Financiera y Actuarial, \\ UB Avenida Diagonal 690, E-08034 Barcelona, Spain \\ Miguel C. Muñoz-Lecanda ${ }^{\text {() }}$ and Narciso Román-Roy ${ }^{\text {d) }}$ \\ Departamento de Matemática Aplicada IV, Edificio C-3, Campus Norte UPC, \\ C/Jordi Girona 1, E-08034 Barcelona, Spain
}

(Received 27 November 2002; accepted 15 September 2003)

The Rusk-Skinner formalism was developed in order to give a geometrical unified formalism for describing mechanical systems. It incorporates all the characteristics of Lagrangian and Hamiltonian descriptions of these systems (including dynamical equations and solutions, constraints, Legendre map, evolution operators, equivalence, etc.). In this work we extend this unified framework to first-order classical field theories, and show how this description comprises the main features of the Lagrangian and Hamiltonian formalisms, both for the regular and singular cases. This formulation is a first step toward further applications in optimal control theory for partial differential equations. (C) 2004 American Institute of Physics.

[DOI: $10.1063 / 1.1628384]$

\section{INTRODUCTION}

In ordinary autonomous classical theories in mechanics there is a unified formulation of Lagrangian and Hamiltonian formalisms, ${ }^{1}$ which is based on the use of the Whitney sum of the tangent and cotangent bundles $W=T Q \oplus T^{*} Q \equiv T Q \times{ }_{Q} T^{*} Q$ (the velocity and momentum phase spaces of the system). In this space, velocities and momenta are independent coordinates. There is a canonical presymplectic form $\Omega$ (the pull-back of the canonical form in $T^{*} Q$ ), and a natural coupling function, locally expressed as $p_{i} v^{i}$, is defined by contraction between vectors and covectors. Given a Lagrangian $L \in C^{\infty}(T Q)$, a Hamiltonian function, locally given by $H=p_{i} v^{i}$ $-L(q, v)$, is determined, and, using the usual constraint algorithm for the geometric equation $i(X) \Omega=\mathrm{d} H$ associated to the Hamiltonian system $(W, \Omega, H)$, we obtain that

(1) The first constraint submanifold $W_{1}$ is isomorphic to $T Q$, and the momenta $\partial L / \partial v^{i}=p_{i}$ are determined as constraints.

(2) The geometric equation contains the second order condition $v^{i}=d q^{i} / d t$.

(3) The identification $W_{1} \equiv T Q$ allows us to recover the Lagrangian formalism.

(4) The projection to the cotangent bundle generates the Hamiltonian formalism, including constraints. The Legendre map and the time evolution operator are straightforwardly obtained by the previous identification and projection. ${ }^{2}$

\footnotetext{
a)Electronic mail: carlos.lopez@uah.es

${ }^{b)}$ Electronic mail: jmarin@ub.edu

${ }^{c}$ Electronic mail: matmcml@mat.upc.es

${ }^{\mathrm{d})}$ Electronic mail: matnrr@mat.upc.es
} 
It is also worth noticing that this space is also appropriate for the formulation of different kinds of problems in optimal control. ${ }^{3-7}$ Furthermore, in Refs. 8 and 9 this unified formalism has been extended for nonautonomous mechanical systems.

Our aim in this paper is to reproduce the same construction for first-order field theories, generating a unified description of Lagrangian and Hamiltonian formalisms and its correspondence, starting from the multisymplectic description of such theories. (See, for instance, Refs. 10-18, for some general references on this formalism. See, also, Refs. 19-25, for other geometric formulations of field theories.) As is shown throughout the paper, characteristics analogous to those pointed out for mechanical systems can be stated in this context. In Ref. 9, a first approach to this subject has been made, focusing mainly on the constraint algorithm for the singular case.

The organization of the paper is as follows: Sec. II is devoted to reviewing the main features of the multisymplectic description of Lagrangian and Hamiltonian field theories. In Sec. III we develop the unified formalism for field theories: starting from the extended jet-multimomentum bundle (analogous to the Whitney sum in mechanics), we introduce the so-called extended Hamiltonian system and state the field equations for sections, $m$-vector fields, connections, and jet fields in this framework. It is also shown how the standard Lagrangian and Hamiltonian descriptions are recovered from this unified picture. As a typical example, the minimal surface problem is described in this formalism in Sec. IV. Finally, we include an Appendix where basic features about connections, jet fields, and $m$-vector fields are displayed.

Throughout this paper $\pi: E \rightarrow M$ will be a fiber bundle $(\operatorname{dim} M=m, \operatorname{dim} E=N+m)$, where $M$ is an oriented manifold with volume form $\omega \in \Omega^{m}(M) . \pi^{1}: J^{1} E \rightarrow E$ is the jet bundle of local sections of $\pi$, and $\bar{\pi}^{1}=\pi^{\circ} \pi^{1}: J^{1} E \rightarrow M$ gives another fiber bundle structure. $\left(x^{\alpha}, y^{A}, v_{\alpha}^{A}\right)$ will denote natural local systems of coordinates in $J^{1} E$, adapted to the bundle $E \rightarrow M(\alpha=1, \ldots, m ; A$ $=1, \ldots, N)$, and such that $\omega=\mathrm{d} x^{1} \wedge \cdots \wedge \mathrm{d} x^{m} \equiv \mathrm{d}^{m} x$. Manifolds are real, paracompact, connected, and $C^{\infty}$. Maps are $C^{\infty}$. Sum over crossed repeated indices is understood.

\section{GEOMETRIC FRAMEWORK FOR CLASSICAL FIELD THEORIES}

\section{A. Lagrangian formalism}

(For details concerning the contents of this and the next section, see, for instance, Refs. 10-13, 17, 18, and 26-31. See, also, the Appendix).

A classical field theory is described by giving a configuration fiber bundle $\pi: E \rightarrow M$ and a Lagrangian density, which is a $\bar{\pi}^{1}$-semibasic $m$-form on $J^{1} E$ usually written as $\mathcal{L}=L \bar{\pi}^{1 *} \omega$, where $L \in C^{\infty}\left(J^{1} E\right)$ is the Lagrangian function determined by $\mathcal{L}$ and $\omega$. The Poincaré-Cartan $m$ and $(m+1)$-forms associated with the Lagrangian density $\mathcal{L}$ are defined using the vertical endomorphism $\mathcal{V}$ of the bundle $J^{1} E$ (see Ref. 30)

$$
\Theta_{\mathcal{L}}:=i(\mathcal{V}) \mathcal{L}+\mathcal{L} \in \Omega^{m}\left(J^{1} E\right) ; \quad \Omega_{\mathcal{L}}:=-\mathrm{d} \Theta_{\mathcal{L}} \in \Omega^{m+1}\left(J^{1} E\right) .
$$

A Lagrangian system is a couple $\left(J^{1} E, \Omega_{\mathcal{L}}\right)$. It is regular if $\Omega_{\mathcal{L}}$ is a multisymplectic $(m+1)$-form (a closed $m$-form, $m>1$, is called multisymplectic if it is one-nondegenerate; elsewhere it is pre-multisymplectic). In natural charts in $J^{1} E$ we have

$$
\mathcal{V}=\left(\mathrm{d} y^{A}-v_{\alpha}^{A} \mathrm{~d} x^{\alpha}\right) \otimes \frac{\partial}{\partial v_{\nu}^{A}} \otimes \frac{\partial}{\partial x^{\nu}},
$$

and

$$
\Theta_{\mathcal{L}}=\frac{\partial L}{\partial v_{\mu}^{A}} \mathrm{~d} y^{A} \wedge \mathrm{d}^{m-1} x_{\mu}-\left(\frac{\partial L}{\partial v_{\mu}^{A}} v_{\mu}^{A}-L\right) \mathrm{d}^{m} x,
$$




$$
\begin{aligned}
\Omega_{\mathcal{L}}= & -\frac{\partial^{2} L}{\partial v_{\nu}^{B} \partial v_{\alpha}^{A}} \mathrm{~d} v_{\nu}^{B} \wedge \mathrm{d} y^{A} \wedge \mathrm{d}^{m-1} x_{\alpha}-\frac{\partial^{2} L}{\partial y^{B} \partial v_{\alpha}^{A}} \mathrm{~d} y^{B} \wedge \mathrm{d} y^{A} \wedge \mathrm{d}^{m-1} x_{\alpha}+\frac{\partial^{2} L}{\partial v_{\nu}^{B} \partial v_{\alpha}^{A}} v_{\alpha}^{A} \mathrm{~d} v_{\nu}^{B} \wedge \mathrm{d}^{m} x \\
& +\left(\frac{\partial^{2} L}{\partial y^{B} \partial v_{\alpha}^{A}} v_{\alpha}^{A}-\frac{\partial L}{\partial y^{B}}+\frac{\partial^{2} L}{\partial x^{\alpha} \partial v_{\alpha}^{B}}\right) \mathrm{d} y^{B} \wedge \mathrm{d}^{m} x
\end{aligned}
$$

(where $\left.\mathrm{d}^{m-1} x_{\alpha} \equiv i\left(\partial / \partial x^{\alpha}\right) \mathrm{d}^{m} x\right)$; the regularity condition is equivalent to $\operatorname{det}\left(\partial^{2} L / \partial v_{\alpha}^{A} \partial v_{\nu}^{B}(\bar{y})\right) \neq 0$, for every $\bar{y} \in J^{1} E$.

The Lagrangian problem associated with a Lagrangian system $\left(J^{1} E, \Omega_{\mathcal{L}}\right)$ consists in finding sections $\phi \in \Gamma(M, E)$, the set of sections of $\pi$, which are characterized by the condition

$$
\left(j^{1} \phi\right)^{*} i(X) \Omega_{L}=0, \quad \text { for every } X \in \mathfrak{X}\left(J^{1} E\right) .
$$

In natural coordinates, if $\phi(x)=\left(x^{\alpha}, \phi^{A}(x)\right)$, this condition is equivalent to demanding that $\phi$ satisfy the Euler-Lagrange equations

$$
\left.\frac{\partial L}{\partial y^{A}}\right|_{j^{1} \phi}-\left.\frac{\partial}{\partial x^{\alpha}}\left(\frac{\partial L}{\partial v_{\alpha}^{A}}\right)\right|_{j^{1} \phi}=0 \quad(\text { for } A=1, \ldots, N)
$$

The problem of finding these sections can be formulated equivalently as follows: finding a distribution $D$ of $T\left(J^{1} E\right)$ such that it is integrable (that is, involutive), $m$-dimensional, $\bar{\pi}^{1}$-transverse, and the integral manifolds of $D$ are the image of sections solution of the above equations (therefore, lifting of $\pi$-sections). This is equivalent to stating that the sections solution to the Lagrangian problem are the integral sections of one of the following equivalent elements:

- A class of holonomic $m$-vector fields $\left\{X_{\mathcal{L}}\right\} \subset \mathfrak{X}^{m}\left(J^{1} E\right)$, such that $i\left(X_{\mathcal{L}}\right) \Omega_{\mathcal{L}}=0$, for every $X_{\mathcal{L}} \in\left\{X_{\mathcal{L}}\right\}$.

- A holonomic connection $\nabla_{\mathcal{L}}$ in $\bar{\pi}^{1}: J^{1} E \rightarrow M$ such that $i\left(\nabla_{\mathcal{L}}\right) \Omega_{\mathcal{L}}=(m-1) \Omega_{\mathcal{L}}$.

- A holonomic jet field $\Psi_{\mathcal{L}}: J^{1} E \rightarrow J^{1} J^{1} E$, such that $i\left(\Psi_{\mathcal{L}}\right) \Omega_{\mathcal{L}}=0$ (the contraction of jet fields with differential forms is defined in Ref. 11).

Semi-holonomic locally decomposable $m$-vector fields, jet fields, and connections which are solution to these equations are called Euler-Lagrange m-vector fields, jet fields, and connections for $\left(J^{1} E, \Omega_{\mathcal{L}}\right)$. In a natural chart in $J^{1} E$, the local expressions of these elements are

$$
\begin{gathered}
X_{\mathcal{L}}=f \underset{\alpha=1}{\wedge}\left(\frac{\partial}{\partial x^{\alpha}}+F_{\alpha}^{A} \frac{\partial}{\partial y^{A}}+G_{\alpha \nu}^{A} \frac{\partial}{\partial v_{\nu}^{A}}\right), \\
\nabla_{\mathcal{L}}=\mathrm{d} x^{\alpha} \otimes\left(\frac{\partial}{\partial x^{\alpha}}+F_{\alpha}^{A} \frac{\partial}{\partial y^{A}}+G_{\alpha \nu}^{A} \frac{\partial}{\partial v_{\nu}^{A}}\right), \\
\Psi_{\mathcal{L}}=\left(x^{\alpha}, y^{A}, v_{\alpha}^{A}, F_{\alpha}^{A}, G_{\alpha \eta}^{A}\right),
\end{gathered}
$$

with $F_{\alpha}^{A}=v_{\alpha}^{A}$ (which is the local expression of the semi-holonomy condition), and where the coefficients $G_{\alpha \nu}^{A}$ are related by the system of linear equations

$$
\frac{\partial^{2} L}{\partial v_{\alpha}^{A} \partial v_{\nu}^{B}} G_{\alpha \nu}^{A}=\frac{\partial L}{\partial y^{B}}-\frac{\partial^{2} L}{\partial x^{\nu} \partial v_{\nu}^{B}}-\frac{\partial^{2} L}{\partial y^{A} \partial v_{\nu}^{B}} v_{\nu}^{A} \quad(A, B=1, \ldots, N)
$$


$f \in C^{\infty}\left(J^{1} E\right)$ is an arbitrary nonvanishing function. A representative of the class $\left\{X_{\mathcal{L}}\right\}$ can be selected by the condition $i\left(X_{\mathcal{L}}\right)\left(\bar{\pi}^{1 *} \omega\right)=1$, which leads to $f=1$ in the above local expression. Therefore, if $j^{1} \phi=\left(x^{\mu}, \phi^{A}, \partial \phi^{A} / \partial x^{\nu}\right)$ is an integral section of $X_{\mathcal{L}}$, then $v_{\alpha}^{A}=\partial \phi^{A} / \partial x^{\alpha}$, and hence, the coefficients $G_{\alpha \nu}^{B}$ must satisfy the equations

$$
G_{\nu \eta}^{A}\left(x^{\alpha}, \phi^{A}, \frac{\partial \phi^{A}}{\partial x^{\alpha}}\right)=\frac{\partial^{2} \phi^{A}}{\partial x^{\eta} \partial x^{\nu}} \quad(A=1, \ldots, N ; \quad \eta, \nu=1, \ldots, m) .
$$

As a consequence, the system (2) is equivalent to the Euler-Lagrange Eq. (1) for $\phi$.

If $\left(J^{1} E, \Omega_{\mathcal{L}}\right)$ is a regular Lagrangian system, the existence of classes of Euler-Lagrange $m$-vector fields for $\mathcal{L}$ (or what is equivalent, Euler-Lagrange jet fields or connections) is assured. For singular Lagrangian systems, the existence of this kind of solutions is not assured except perhaps on some submanifold $S \hookrightarrow J^{1} E$. Furthermore, solutions of the field equations can exist (in general, on some submanifold of $J^{1} E$ ), but none of them are semi-holonomic (at any point of this submanifold). In both cases, the integrability of these solutions is not assured, except perhaps on a smaller submanifold $I$ such that the integral sections are contained in $I$.

\section{B. Hamiltonian formalism}

For the Hamiltonian formalism of field theories, we have the extended multimomentum bundle $\mathcal{M} \pi$, which is the bundle of $m$-forms on $E$ vanishing by contraction with two $\pi$-vertical vector fields [or equivalently, the set of affine maps from $J^{1} E$ to $\pi^{*} \Lambda^{m} T^{*} M$ (Refs. 10 and 32)], and the restricted multimomentum bundle $J^{1 *} E \equiv \mathcal{M} \pi / \pi^{*} \Lambda^{m} T^{*} M$. We have the natural projections

$$
\tau^{1}: J^{1 *} E \rightarrow E, \quad \bar{\tau}^{1}=\pi^{\circ} \tau^{1}: J^{1 *} E \rightarrow M, \quad \mu: \mathcal{M} \pi \rightarrow J^{1 *} E, \quad \hat{\mu}=\bar{\tau}^{1} \circ \mu: \mathcal{M} \pi \rightarrow M .
$$

Given a system of coordinates adapted to the bundle $\pi: E \rightarrow M$, we can construct natural coordinates $\left(x^{\alpha}, y^{A}, p_{A}^{\alpha}, p\right)(\alpha=1, \ldots, m ; A=1, \ldots, N)$ in $\mathcal{M} \pi$, corresponding to the $m$-covector $\mathbf{p}=p \mathrm{~d}^{m} x$ $+p_{A}^{\alpha} \mathrm{d} y^{A} \wedge \mathrm{d}^{m-1} x_{\alpha} \in \mathcal{M} \pi$, and $\left(x^{\alpha}, y^{A}, p_{A}^{\alpha}\right)$ in $J^{1 *} E$, for the class [p] $=p_{A}^{\alpha} \mathrm{d} y^{A} \wedge \mathrm{d}^{m-1} x_{\alpha}+\left\langle\mathrm{d}^{m} x\right\rangle$ $\in J^{1 *} E$.

Now, if $\left(J^{1} E, \Omega_{\mathcal{L}}\right)$ is a Lagrangian system, the extended Legendre map associated with $\mathcal{L}$, $\widetilde{\mathcal{F} L}: J^{1} E \rightarrow \mathcal{M} \pi$, is defined as

$$
[\widetilde{\mathcal{F} L}(\bar{y})]\left(Z_{1}, \ldots, Z_{m}\right):=\left(\Theta_{\mathcal{L}}\right)_{\bar{y}}\left(\bar{Z}_{1}, \ldots, \bar{Z}_{m}\right),
$$

where $Z_{1}, \ldots, Z_{m} \in T_{\pi^{1}(\bar{y})} E$, and $\bar{Z}_{1}, \ldots, \bar{Z}_{m} \in T_{\bar{y}} J^{1} E$ are such that $T_{\bar{y}} \pi^{1} \bar{Z}_{\alpha}=Z_{\alpha}$. Then the restricted Legendre map associated with $\mathcal{L}$ is $\mathcal{F} \mathcal{L}:=\mu \circ \widetilde{\mathcal{F} L}$. Their local expressions are

$$
\begin{aligned}
& \widetilde{\mathcal{F}}^{*} x^{\alpha}=x^{\alpha}, \quad \widetilde{\mathcal{F} \mathcal{L}^{*}} y^{A}=y^{A}, \quad \widetilde{\mathcal{F} \mathcal{L}^{*}} p_{A}^{\alpha}=\frac{\partial L}{\partial v_{\alpha}^{A}}, \quad \widetilde{\mathcal{F} \mathcal{L}^{*}} p=L-v_{\alpha}^{A} \frac{\partial L}{\partial v_{\alpha}^{A}}, \\
& \mathcal{F} \mathcal{L}^{*} x^{\alpha}=x^{\alpha}, \quad \mathcal{F} \mathcal{L}^{*} y^{A}=y^{A}, \quad \mathcal{F} \mathcal{L}^{*} p_{A}^{\alpha}=\frac{\partial L}{\partial v_{\alpha}^{A}} .
\end{aligned}
$$

Therefore, $\left(J^{1} E, \Omega_{\mathcal{L}}\right)$ is a regular Lagrangian system if $\mathcal{F L}$ is a local diffeomorphism (this definition is equivalent to that given above). Elsewhere $\left(J^{1} E, \Omega_{\mathcal{L}}\right)$ is a singular Lagrangian system. As a particular case, $\left(J^{1} E, \Omega_{\mathcal{L}}\right)$ is a hyper-regular Lagrangian system if $\mathcal{F} \mathcal{L}$ is a global diffeomorphism. A singular Lagrangian system $\left(J^{1} E, \Omega_{\mathcal{L}}\right)$ is almost-regular if: $\mathcal{P}:=\mathcal{F} \mathcal{L}\left(J^{1} E\right)$ is a closed submanifold of $J^{1 *} E$ (we will denote the natural imbedding by $J: \mathcal{P} \hookrightarrow J^{1 *} E$ ), $\mathcal{F} \mathcal{L}$ is a submersion onto its image, and for every $\bar{y} \in J^{1} E$, the fibres $\mathcal{F} \mathcal{L}^{-1}(\mathcal{F} \mathcal{L}(\bar{y}))$ are connected submanifolds of $J^{1} E$.

In order to construct a Hamiltonian system associated with $\left(J^{1} E, \Omega_{\mathcal{L}}\right)$, recall that the multicotangent bundle $\Lambda^{m} T^{*} E$ is endowed with a natural canonical form $\Theta \in \Omega^{m}\left(\Lambda^{m} T^{*} E\right)$, which is 
the tautological form defined as follows: let $\tau_{E}: T^{*} E \rightarrow E$ be the natural projection, and $\Lambda^{m} \tau_{E}: \Lambda^{m} T^{*} E \rightarrow E$ its natural extension; then, for every $\overline{\mathbf{p}} \in \Lambda^{m} T^{*} E$ (where $\overline{\mathbf{p}}=(y, \beta)$, with $y$ $\in E$ and $\left.\beta \in \Lambda^{m} T_{y}^{*} E\right)$, and for every $X_{1}, \ldots, X_{m} \in \mathfrak{X}\left(\Lambda^{m} T^{*} E\right)$ we have

$$
\left[\boldsymbol{\Theta}\left(X_{1}, \ldots, X_{m}\right)\right]_{\overline{\mathbf{p}}}:=\left[\left(\Lambda^{m} \tau_{E}\right)^{*} \beta\right]\left(X_{1_{\overline{\mathbf{p}}}}, \ldots, X_{m_{\overline{\mathbf{p}}}}\right)=\beta\left(T_{\overline{\mathbf{p}}} \Lambda^{m} \tau_{E}\left(X_{1_{\overline{\mathbf{p}}}}\right), \ldots, T_{\overline{\mathbf{p}}} \Lambda^{m} \tau_{E}\left(X_{m_{\overline{\mathbf{p}}}}\right)\right) .
$$

Thus we also have the multisymplectic form $\boldsymbol{\Omega}:=-\mathrm{d} \boldsymbol{\Theta} \in \Omega^{m+1}\left(\Lambda^{m} T^{*} E\right)$. But $\mathcal{M} \pi \equiv \Lambda_{1}^{m} T^{*} E$ is a subbundle of $\Lambda^{m} T^{*} E$. Then, if $\lambda: \Lambda_{1}^{m} T^{*} E \hookrightarrow \Lambda^{m} T^{*} E$ is the natural imbedding, $\Theta:=\lambda^{*} \Theta$ and $\Omega:=-\mathrm{d} \Theta=\lambda^{*} \boldsymbol{\Omega}$ are canonical forms in $\mathcal{M} \pi$, which are called the multimomentum Liouville $m$ and $(m+1)$ forms. In particular, we have that $\Theta(\mathbf{p})=\left(\tau_{1}{ }^{\circ} \mu\right)^{*} \mathbf{p}$, for every $\mathbf{p} \in \mathcal{M} \pi$. Their local expressions are

$$
\Theta=p_{A}^{\alpha} \mathrm{d} y^{A} \wedge \mathrm{d}^{m-1} x_{\alpha}+p \mathrm{~d}^{m} x, \quad \Omega=-\mathrm{d} p_{A}^{\alpha} \wedge \mathrm{d} y^{A} \wedge \mathrm{d}^{m-1} x_{\alpha}-\mathrm{d} p \wedge \mathrm{d}^{m} x .
$$

Observe that $\widetilde{\mathcal{F}}^{*} \Theta=\Theta_{\mathcal{L}}$, and $\widetilde{\mathcal{F}}^{*} \Omega=\Omega_{\mathcal{L}}$.

Now, if $\left(J^{1} E, \Omega_{\mathcal{L}}\right)$ is a hyper-regular Lagrangian system, then $\tilde{\mathcal{P}}:=\widetilde{\mathcal{F}} \mathcal{L}\left(J^{1} E\right)$ is a onecodimensional and $\mu$-transverse imbedded submanifold of $\mathcal{M} \pi$ (we will denote the natural imbedding by $\tilde{J}: \widetilde{\mathcal{P}} \hookrightarrow \mathcal{M} \pi$ ), which is diffeomorphic to $J^{1 *} E$. This diffeomorphism is $\mu^{-1}$, when $\mu$ is restricted to $\tilde{\mathcal{P}}$, and also coincides with the map $h:=\widetilde{\mathcal{F}} \mathcal{L} \circ \mathcal{F} \mathcal{L}^{-1}$, when it is restricted onto its image (which is just $\tilde{\mathcal{P}}$ ). This map $h$ is called a Hamiltonian section, and can be used to construct the Hamilton-Cartan $m$ and $(m+1)$ forms of $J^{1 *} E$ by making

$$
\Theta_{h}=h^{*} \Theta \in \Omega^{m}\left(J^{1 *} E\right), \quad \Omega_{h}=h^{*} \Omega \in \Omega^{m+1}\left(J^{1 *} E\right) .
$$

The couple $\left(J^{1 * E}, \Omega_{h}\right)$ is said to be the Hamiltonian system associated with the hyper-regular Lagrangian system $\left(J^{1} E, \Omega_{\mathcal{L}}\right)$. Locally, the Hamiltonian section $h$ is specified by the local Hamiltonian function $H=p_{A}^{\alpha}\left(F \mathcal{L}^{-1}\right)^{*} v_{\alpha}^{A}-\left(F \mathcal{L}^{-1}\right) * L$, that is, $h\left(x^{\alpha}, y^{A}, p_{A}^{\alpha}\right)=\left(x^{\alpha}, y^{A}, p_{A}^{\alpha},-H\right)$. Then we have the local expressions

$$
\Theta_{h}=p_{A}^{\alpha} \mathrm{d} y^{A} \wedge \mathrm{d}^{m-1} x_{\alpha}-H \mathrm{~d}^{m} x, \quad \Omega_{h}=-\mathrm{d} p_{A}^{\alpha} \wedge \mathrm{d} y^{A} \wedge \mathrm{d}^{m-1} x_{\alpha}+\mathrm{d} H \wedge \mathrm{d}^{m} x
$$

Of course $\mathcal{F} \mathcal{L}^{*} \Theta_{h}=\Theta_{\mathcal{L}}$ and $\mathcal{F} \mathcal{L}^{*} \Omega_{h}=\Omega_{\mathcal{L}}$.

The Hamiltonian problem associated with the Hamiltonian system $\left(J^{1 *} E, \Omega_{h}\right)$ consists in finding sections $\psi \in \Gamma\left(M, J^{1 *} E\right)$, which are characterized by the condition

$$
\psi^{*} i(X) \Omega_{h}=0, \text { for every } X \in \mathfrak{X}\left(J^{1 *} E\right) .
$$

In natural coordinates, if $\psi(x)=\left(x^{\alpha}, y^{A}(x), p_{A}^{\alpha}(x)\right)$, this condition leads to the so-called Hamilton-De Donder-Weyl equations (for the section $\psi$ ).

The problem of finding these sections can be formulated equivalently as follows: finding a distribution $D$ of $T\left(J^{1 *} E\right)$ such that $D$ is integrable (that is, involutive), $m$-dimensional, $\bar{\tau}^{1}$-transverse, and its integral manifolds are the sections solution to the above equations. This is equivalent to stating that the sections solution to the Hamiltonian problem are the integral sections of one of the following equivalent elements:

- A class of integrable and $\bar{\tau}^{1}$-transverse $m$-vector fields $\left\{X_{\mathcal{H}}\right\} \subset \mathfrak{X}^{m}\left(J^{1 *} E\right)$ satisfying that $i\left(X_{\mathcal{H}}\right) \Omega_{h}=0$, for every $X_{\mathcal{H}} \in\left\{X_{\mathcal{H}}\right\}$.

- An integrable connection $\nabla_{\mathcal{H}}$ in $\bar{\tau}^{1}: J^{1 *} E \rightarrow M$ such that $i\left(\nabla_{\mathcal{H}}\right) \Omega_{h}=(m-1) \Omega_{h}$.

- An integrable jet field $\Psi_{\mathcal{H}}: J^{1 *} E \rightarrow J^{1} J^{1 *} E$, such that $i\left(\Psi_{\mathcal{H}}\right) \Omega_{h}=0$.

$\bar{\tau}^{1}$-transverse and locally decomposable $m$-vector fields, orientable jet fields, and orientable connections, which are solutions of these equations, are called Hamilton-De Donder-Weyl (HDW) $m$-vector fields, jet fields, and connections for $\left(J^{1 *} E, \Omega_{h}\right)$. Their local expressions in natural coordinates are 


$$
\begin{gathered}
X_{\mathcal{H}}=\underset{\alpha=1}{f \wedge}\left(\frac{\partial}{\partial x^{\alpha}}+F_{\alpha}^{A} \frac{\partial}{\partial y^{A}}+G_{A \alpha}^{\eta} \frac{\partial}{\partial p_{A}^{\eta}}\right), \\
\Psi_{\mathcal{H}}=\left(x^{\alpha}, y^{A}, p_{A}^{\alpha} ; F_{\alpha}^{A}, G_{A \alpha}^{\eta}\right), \\
\nabla_{\mathcal{H}}=\mathrm{d} x^{\alpha} \otimes\left(\frac{\partial}{\partial x^{\alpha}}+F_{\alpha}^{A} \frac{\partial}{\partial y^{A}}+G_{A \alpha}^{\nu} \frac{\partial}{\partial p_{A}^{\nu}}\right),
\end{gathered}
$$

where $f \in C^{\infty}\left(J^{1 *} E\right)$ is a nonvanishing function, and the coefficients $F_{\alpha}^{A}, G_{A \alpha}^{\eta}$ are related by the system of linear equations

$$
F_{\alpha}^{A}=\frac{\partial H}{\partial p_{A}^{\alpha}}, \quad G_{A \nu}^{\nu}=-\frac{\partial H}{\partial y^{A}} .
$$

Now, if $\psi(x)=\left(x^{\alpha}, y^{A}(x)=\psi^{A}(x), p_{A}^{\alpha}(x)=\psi_{A}^{\alpha}(x)\right)$ is an integral section of $X_{\mathcal{H}}$ then

$$
\left.\frac{\partial H}{\partial p_{A}^{\alpha}}\right|_{\psi}=F_{\alpha}^{A}{ }_{\alpha} \psi=\frac{\partial \psi^{A}}{\partial x_{\alpha}} ; \quad-\left.\frac{\partial H}{\partial y^{A}}\right|_{\psi}=G_{A \alpha}^{\alpha}{ }^{\circ} \psi=\frac{\partial \psi_{A}^{\alpha}}{\partial x^{\alpha}},
$$

which are the Hamilton-De Donder-Weyl equations for $\psi$. As above, a representative of the class $\left\{X_{\mathcal{H}}\right\}$ can be selected by the condition $i\left(X_{\mathcal{H}}\right)\left(\bar{\tau}^{1 *} \omega\right)=1$, which leads to $f=1$ in the above local expression. The existence of classes of HDW $m$-vector fields, jet fields, and connections is assured.

In an analogous way, if $\left(J^{1} E, \Omega_{\mathcal{L}}\right)$ is an almost-regular Lagrangian system, the submanifold $J: \mathcal{P} \hookrightarrow J^{1 *} E$, is a fiber bundle over $E$ and $M$. In this case the $\mu$-transverse submanifold $\widetilde{\mathcal{P}} \mathcal{M} \pi$ is diffeomorphic to $\mathcal{P}$. This diffeomorphism is denoted by $\tilde{\mu}: \widetilde{\mathcal{P}} \rightarrow \mathcal{P}$, and it is just the restriction of the projection $\mu$ to $\widetilde{\mathcal{P}}$. Then, taking the Hamiltonian section $\tilde{h}:=\tilde{J}^{\circ} \tilde{\mu}^{-1}$, we define the HamiltonCartan forms

$$
\Theta_{h}^{0}=\tilde{h}^{*} \Theta ; \quad \Omega_{h}^{0}=\tilde{h}^{*} \Omega
$$

which verify that $\mathcal{F} \mathcal{L}_{0}^{*} \Theta_{h}^{0}=\Theta_{\mathcal{L}}$ and $\mathcal{F} \mathcal{L}_{0}^{*} \Theta_{h}^{0}=\Omega_{\mathcal{L}}$ (where $\mathcal{F} \mathcal{L}_{0}$ is the restriction map of $\mathcal{F} \mathcal{L}$ onto $\mathcal{P})$. Then $\left(\mathcal{P}, \Omega_{h}^{0}\right)$ is the Hamiltonian system associated with the almost-regular Lagrangian system $\left(J^{1} E, \Omega_{\mathcal{L}}\right)$, and we have Diagram 1.

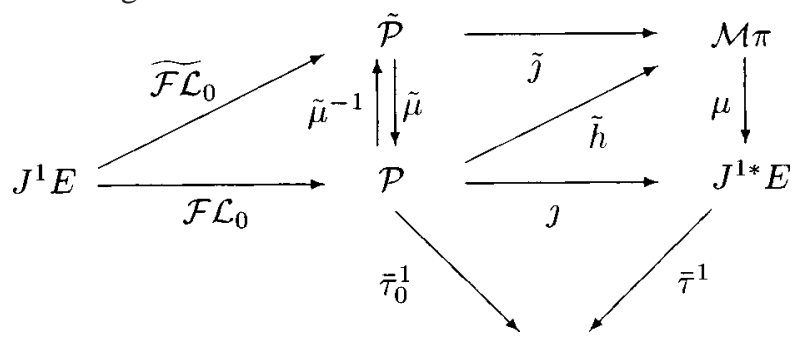

$M$

Then, the Hamiltonian problem associated with the Hamiltonian system $\left(\mathcal{P}, \Omega_{h}^{0}\right)$, and the equations for the sections of $\Gamma(M, \mathcal{P})$ solution to the Hamiltonian problem are stated as in the regular case. Now, the existence of the corresponding Hamilton-De Donder-Weyl $m$-vector fields, jet fields, and connections for $\left(\mathcal{P}, \Omega_{h}^{0}\right)$ is not assured, except perhaps on some submanifold $P$ of $\mathcal{P}$, where the solution is not unique.

From now on we will consider only regular or almost-regular systems. 


\section{UNIFIED FORMALISM}

\section{A. Extended Hamiltonian system}

Given a fiber bundle $\pi: E \rightarrow M$ over an oriented manifold $(M, \omega)$, we define the extended jet-multimomentum bundle $\mathcal{W}$ and the restricted jet-multimomentum bundle $\mathcal{W}_{r}$ as

$$
\mathcal{W}:=J^{1} E \times_{E} \mathcal{M} \pi, \quad \mathcal{W}_{r}:=J^{1} E \times_{E} J^{1 *} E,
$$

whose natural coordinates are $\left(x^{\alpha}, y^{A}, v_{\alpha}^{A}, p_{A}^{\alpha}, p\right)$ and $\left(x^{\alpha}, y^{A}, v_{\alpha}^{A}, p_{A}^{\alpha}\right)$, respectively. We have the natural projections (submersions)

$$
\begin{aligned}
& \rho_{1}: \mathcal{W} \rightarrow J^{1} E, \quad \rho_{2}: \mathcal{W} \rightarrow \mathcal{M} \pi, \quad \rho_{E}: \mathcal{W} \rightarrow E, \quad \rho_{M}: \mathcal{W} \rightarrow M, \\
& \rho_{1}^{r}: \mathcal{W}_{r} \rightarrow J^{1} E, \quad \rho_{2}^{r}: \mathcal{W}_{r} \rightarrow J^{1 *} E, \quad \rho_{E}^{r}: \mathcal{W}_{r} \rightarrow E, \quad \rho_{M}^{r}: \mathcal{W}_{r} \rightarrow M .
\end{aligned}
$$

Note that $\pi^{1} \circ \rho_{1}=\tau^{1} \circ \mu^{\circ} \rho_{2}=\rho_{E}$. In addition, there is also the natural projection

$$
\begin{gathered}
\mu_{\mathcal{W}}: \mathcal{W} \rightarrow \mathcal{W}_{r}, \\
(\bar{y}, \mathbf{p}) \mapsto(\bar{y},[\mathbf{p}]) .
\end{gathered}
$$

The bundle $\mathcal{W}$ is endowed with the following canonical structures:

\section{Definition 1:}

(1) The coupling $m$-form in $\mathcal{W}$, denoted by $\mathcal{C}$, is an $m$-form along $\rho_{M}$ which is defined as follows: for every $\bar{y} \in J_{y}^{1} E$, with $\bar{\pi}^{1}(\bar{y})=\pi(y)=x \in E$, and $\mathbf{p} \in \mathcal{M}_{y} \pi$, let $w \equiv(\bar{y}, \mathbf{p}) \in \mathcal{W}_{y}$, then

$$
\mathcal{C}(w):=\left(T_{x} \phi\right)^{*} \mathbf{p},
$$

where $\phi: M \rightarrow E$ satisfies that $j^{1} \phi(x)=\bar{y}$.

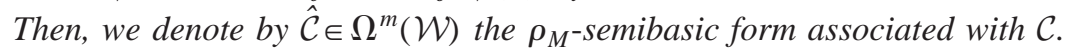

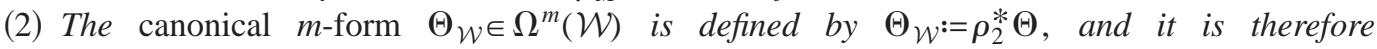
$\rho_{E^{-}}$semibasic.

The canonical $(m+1)$-form is the pre-multisymplectic form $\Omega_{\mathcal{W}}:=-\mathrm{d} \Theta_{\mathcal{W}}=\rho_{1}^{*} \Omega$ $\in \Omega^{m+1}(\mathcal{W})$.

Being $\hat{\mathcal{C}}$ a $\rho_{M}$-semibasic form, there is $\hat{C} \in C^{\infty}(\mathcal{W})$ such that $\hat{\mathcal{C}}=\hat{C}\left(\rho_{M}^{*} \omega\right)$. Note also that $\Omega_{\mathcal{W}}$ is not one-nondegenerate, its kernel being the $\rho_{2}$-vertical vectors; then, we call $\left(\mathcal{W}, \Omega_{\mathcal{W}}\right)$ a premultisymplectic structure. This definition of the coupling form is in fact an alternative (obviously equivalent) presentation of the extended multimomentum bundle as the set of affine maps from the jet bundle $J^{1} E$ to $\pi$-basic $m$-forms.

The local expressions for $\Theta_{\mathcal{W}}$ and $\Omega_{\mathcal{W}}$ are the same as (4), and for $\hat{\mathcal{C}}$ we have

$$
\hat{\mathcal{C}}(w)=\left(p+p_{A}^{\alpha} v_{\alpha}^{A}\right) \mathrm{d}^{m} x .
$$

Given a Lagrangian density $\mathcal{L} \in \Omega^{m}\left(J^{1} E\right)$, we denote $\hat{\mathcal{L}}:=\rho_{1}^{*} \mathcal{L} \in \Omega^{m}(\mathcal{W})$, and we can write $\hat{\mathcal{L}}=\hat{L}\left(\rho_{M}^{*} \omega\right)$, with $\hat{L}=\rho_{1}^{*} L \in C^{\infty}(\mathcal{W})$. We define a Hamiltonian submanifold

$$
\mathcal{W}_{0}:=\{w \in \mathcal{W} \mid \hat{\mathcal{L}}(w)=\hat{\mathcal{C}}(w)\} .
$$

So, $\mathcal{W}_{0}$ is the submanifold of $\mathcal{W}$ defined by the constraint function $\hat{C}-\hat{L}=0$. In local coordinates this constraint function is

$$
p+p_{A}^{\alpha} v_{\alpha}^{A}-\hat{L}\left(x^{\nu}, y^{B}, v_{\nu}^{B}\right)=0 .
$$


We have the natural imbedding $J_{0}: \mathcal{W}_{0} \hookrightarrow \mathcal{W}$, as well as the projections (submersions)

$$
\rho_{1}^{0}: \mathcal{W}_{0} \rightarrow J^{1} E, \quad \rho_{2}^{0}: \mathcal{W}_{0} \rightarrow \mathcal{M} \pi, \quad \rho_{E}^{0}: \mathcal{W}_{0} \rightarrow E, \quad \rho_{M}^{0}: \mathcal{W}_{0} \rightarrow M
$$

which are the restrictions to $\mathcal{W}_{0}$ of the projections (6), and $\hat{\rho}_{2}^{0}=\mu^{\circ} \rho_{2}^{0}: \mathcal{W}_{0} \rightarrow J^{1 *} E$. So we have Diagram 2.

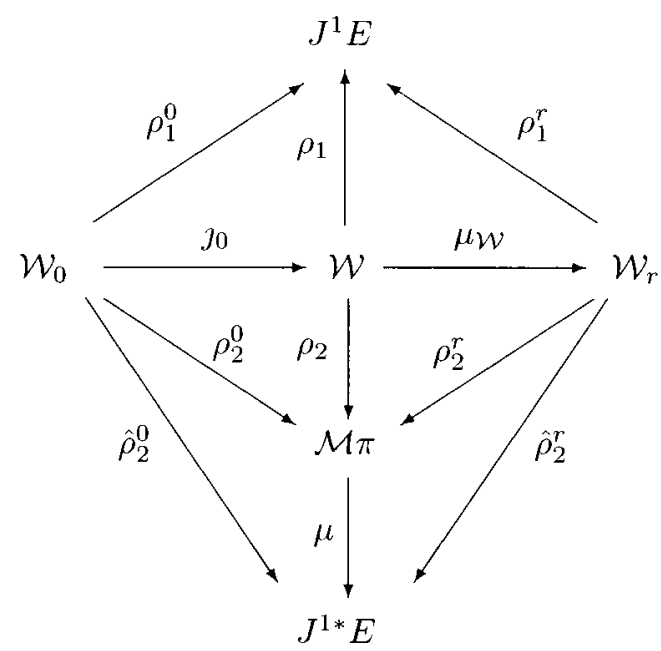

Local coordinates in $\mathcal{W}_{0}$ are $\left(x^{\alpha}, y^{A}, v_{\alpha}^{A}, p_{A}^{\alpha}\right)$, and we have that

$$
\begin{gathered}
\rho_{1}^{0}\left(x^{\alpha}, y^{A}, v_{\alpha}^{A}, p_{A}^{\alpha}\right)=\left(x^{\alpha}, y^{A}, v_{\alpha}^{A}\right), \\
J_{0}\left(x^{\alpha}, y^{A}, v_{\alpha}^{A}, p_{A}^{\alpha}\right)=\left(x^{\alpha}, y^{A}, v_{\alpha}^{A}, p_{A}^{\alpha}, L-v_{\alpha}^{A} p_{A}^{\alpha}\right), \\
\rho_{2}^{0}\left(x^{\alpha}, y^{A}, v_{\alpha}^{A}, p_{A}^{\alpha}\right)=\left(x^{\alpha}, y^{A}, p_{A}^{\alpha}, L-v_{\alpha}^{A} p_{A}^{\alpha}\right), \\
\hat{\rho}_{2}^{0}\left(x^{\alpha}, y^{A}, v_{\alpha}^{A}, p_{A}^{\alpha}\right)=\left(x^{\alpha}, y^{A}, p_{A}^{\alpha}\right) .
\end{gathered}
$$

Proposition 1: $\mathcal{W}_{0}$ is a one-codimensional $\mu_{\mathcal{W}}$-transversal submanifold of $\mathcal{W}$, diffeomorphic to $\mathcal{W}_{r}$

(Proof) For every $(\bar{y}, \mathbf{p}) \in \mathcal{W}_{0}, \quad$ we have $L(\bar{y}) \equiv \hat{L}(\bar{y}, \mathbf{p})=\hat{C}(\bar{y}, \mathbf{p})$,

and

$$
\left(\mu_{\mathcal{W}^{\circ} J_{0}}\right)(\bar{y}, \mathbf{p})=\mu_{\mathcal{W}}(\bar{y}, \mathbf{p})=(\bar{y}, \mu(\mathbf{p}))=(\bar{y},[\mathbf{p}])
$$

First, $\mu_{\mathcal{W}^{\circ} J_{0}}$ is injective: let $\left(\bar{y}_{1}, \mathbf{p}_{1}\right),\left(\bar{y}_{2}, \mathbf{p}_{2}\right) \in \mathcal{W}_{0}$, then we have

$$
\left(\mu_{\mathcal{W}^{\circ} J_{0}}\right)\left(\bar{y}_{1}, \mathbf{p}_{1}\right)=\left(\mu_{\mathcal{W}^{\circ} J_{0}}\right)\left(\bar{y}_{2}, \mathbf{p}_{2}\right) \Rightarrow\left(\bar{y}_{1}, \mu\left(\mathbf{p}_{1}\right)\right)=\left(\bar{y}_{2}, \mu\left(\mathbf{p}_{2}\right)\right) \Rightarrow \bar{y}_{1}=\bar{y}_{2}, \mu\left(\mathbf{p}_{1}\right)=\mu\left(\mathbf{p}_{2}\right),
$$

hence,

$$
L\left(\bar{y}_{1}\right)=L\left(\bar{y}_{2}\right)=\hat{C}\left(\bar{y}_{1}, \mathbf{p}_{1}\right)=\hat{C}\left(\bar{y}_{2}, \mathbf{p}_{2}\right) .
$$

In a local chart, third equality gives

$$
p\left(\mathbf{p}_{1}\right)+p_{A}^{\alpha}\left(\mathbf{p}_{1}\right) v_{\alpha}^{A}\left(\bar{y}_{1}\right)=p\left(\mathbf{p}_{2}\right)+p_{A}^{\alpha}\left(\mathbf{p}_{2}\right) v_{\alpha}^{A}\left(\bar{y}_{2}\right),
$$

but $\mu\left(\mathbf{p}_{1}\right)=\mu\left(\mathbf{p}_{2}\right)$ implies that 


$$
p_{A}^{\alpha}\left(\mathbf{p}_{1}\right)=p_{A}^{\alpha}\left(\left[\mathbf{p}_{1}\right]\right)=p_{A}^{\alpha}\left(\left[\mathbf{p}_{2}\right]\right)=p_{A}^{\alpha}\left(\mathbf{p}_{2}\right),
$$

therefore, $p\left(\mathbf{p}_{1}\right)=p\left(\mathbf{p}_{2}\right)$, and hence, $\mathbf{p}_{1}=\mathbf{p}_{2}$.

Second, $\mu_{\mathcal{W}^{\circ} J_{0}}$ is onto: Let $(\bar{y}, \mathbf{p}) \in \mathcal{W}_{r}$, then there exists $(\bar{y}, \mathbf{q}) \in J_{0}\left(\mathcal{W}_{0}\right)$ such that $[\mathbf{q}]=[\mathbf{p}]$. In fact, it suffices to take [q] in such a way that, in a local chart of $J^{1} E \times{ }_{E} \mathcal{M} \pi=\mathcal{W}$

$$
p_{A}^{\alpha}(\mathbf{q})=p_{A}^{\alpha}([\mathbf{p}]), \quad p(\mathbf{q})=p_{A}^{\alpha}([\mathbf{p}]) v_{\alpha}^{A}(\bar{y})-L(\bar{y}) .
$$

Finally, observe that $\mathcal{W}_{0}$ is defined by the constraint function $\hat{L}-\hat{C}$ and, as ker $\mu_{\mathcal{W} *}$ $=\{\partial / \partial p\}$ and $\partial / \partial p(\hat{L}-\hat{C})=1$, then $\mathcal{W}_{0}$ is a 1 -codimensional submanifold of $\mathcal{W}$ and $\mu_{\mathcal{W}}$-transversal.

As a consequence of this property, the submanifold $\mathcal{W}_{0}$ induces a section $\hat{h}: \mathcal{W}_{r} \rightarrow \mathcal{W}$ of the projection $\mu_{\mathcal{W}}$. Locally, $\hat{h}$ is specified by giving the local Hamiltonian function $\hat{H}=-\hat{L}$ $+p_{A}^{\alpha} v_{\alpha}^{A}$; that is, $\hat{h}\left(x^{\alpha}, y^{A}, v_{\alpha}^{A}, p_{A}^{\alpha}\right)=\left(x^{\alpha}, y^{A}, v_{\alpha}^{A}, p_{A}^{\alpha},-\hat{H}\right)$. In this sense, $\hat{h}$ is said to be a Hamiltonian section of $\mu_{\mathcal{W}}$.

Remark:It is important to point out that, from every Hamiltonian $\mu_{\mathcal{W}}$-section $\hat{h}: \mathcal{W}_{r} \rightarrow \mathcal{W}$ in the extended unified formalism, we can recover a Hamiltonian $\mu$-section $\widetilde{h}: \mathcal{P} \rightarrow \mathcal{M} \pi$ in the standard Hamiltonian formalism. In fact, given $[\mathbf{p}] \in J^{1 *} E$, the section $\hat{h}$ maps every point $(\bar{y},[\mathbf{p}])$ $\in\left(\rho_{2}^{r}\right)^{-1}([\mathbf{p}])$ into $\rho_{2}^{-1}\left[\rho_{2}(\hat{h}(\bar{y},[\mathbf{p}]))\right]$. So, the crucial point is the projectability of the local function $\hat{H}$ by $\rho_{2}$. But, being $\partial / \partial v_{\alpha}^{A}$ a local basis for ker $\rho_{2 *}, \hat{H}$ is $\rho_{2}$-projectable if, and only if, $p_{A}^{\alpha}=\partial L / \partial v_{A}^{\alpha}$, and this condition is fulfilled when $[\mathbf{p}] \in \mathcal{P}=\operatorname{Im} \mathcal{F} \mathcal{L} \subset J^{1 *} E$, which implies that $\left.\left.\rho_{2}\left[\hat{h}\left(\rho_{2}^{r}\right)^{-1}\right)([\mathbf{p}])\right)\right] \in \widetilde{\mathcal{P}}=\operatorname{Im} \mathcal{F} \mathcal{L} \subset \mathcal{M} \pi$. Hence, the Hamiltonian section $\widetilde{h}$ is defined as follows:

$$
\bar{h}([\mathbf{p}])=\left(\rho_{2} \circ \hat{h}\right)\left[\left(\rho_{2}^{r}\right)^{-1}(J([\mathbf{p}]))\right], \quad \text { for every }[\mathbf{p}] \in \mathcal{P} .
$$

So we have Diagram 3 (see also Diagram 1).

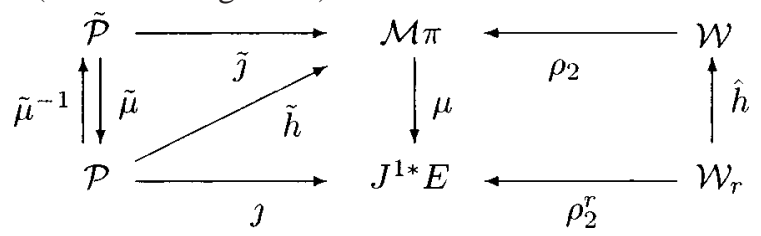

(For (hyper) regular systems this diagram is the same with $\operatorname{Im} \mathcal{F} \mathcal{L}=J^{1 *} E$.)

Finally, we can define the forms

$$
\Theta_{0}:=j_{0}^{*} \Theta_{\mathcal{W}}=\rho_{2}^{0 *} \Theta \in \Omega^{m}\left(\mathcal{W}_{0}\right), \quad \Omega_{0}:=j_{0}^{*} \Omega_{\mathcal{W}}=\rho_{2}^{0 *} \Omega \in \Omega^{m+1}\left(\mathcal{W}_{0}\right),
$$

with local expressions

$$
\begin{gathered}
\Theta_{0}=\left(L-p_{A}^{\alpha} v_{\alpha}^{A}\right) \mathrm{d}^{m} x+p_{A}^{\alpha} \mathrm{d} y^{A} \wedge \mathrm{d}^{m-1} x_{\alpha}, \\
\Omega_{0}=\mathrm{d}\left(p_{A}^{\alpha} v_{\alpha}^{A}-L\right) \wedge \mathrm{d}^{m} x-\mathrm{d} p_{A}^{\alpha} \wedge \mathrm{d} y^{A} \wedge \mathrm{d}^{m-1} x_{\alpha},
\end{gathered}
$$

and we have obtained a (pre-multisymplectic) Hamiltonian system $\left(\mathcal{W}_{0}, \Omega_{0}\right)$, or equivalently $\left(\mathcal{W}_{r}, \hat{h}^{*} \Omega_{\mathcal{W}}\right)$.

\section{B. Field equations for sections}

The Lagrange-Hamiltonian problem associated with the system $\left(\mathcal{W}_{0}, \Omega_{0}\right)$ consists in finding sections $\psi_{0} \in \Gamma\left(M, \mathcal{W}_{0}\right)$ which are characterized by the condition

$$
\psi_{0}^{*} i\left(Y_{0}\right) \Omega_{0}=0, \text { for every } Y_{0} \in \mathfrak{X}\left(\mathcal{W}_{0}\right) .
$$


This equation gives different kinds of information, depending on the type of the vector fields $Y_{0}$ involved. In particular, using vector fields $Y_{0}$ which are $\hat{\rho}_{2}^{0}$-vertical, we have:

Lemma 1: If $Y_{0} \in \mathfrak{X}^{V\left(\hat{\rho}_{2}^{0}\right)}\left(\mathcal{W}_{0}\right)$ (i.e., $Y_{0}$ is $\hat{\rho}_{2}^{0}$-vertical), then $i\left(Y_{0}\right) \Omega_{0}$ is $\rho_{M}^{0}$-semibasic.

(Proof) A simple calculation in coordinates leads to this result. In fact, taking $\left\{\partial / \partial v_{\alpha}^{A}\right\}$ as a local basis for the $\hat{\rho}_{2}^{0}$-vertical vector fields, and bearing in mind (7) we obtain

$$
i\left(\frac{\partial}{\partial v_{\alpha}^{A}}\right) \Omega_{0}=\left(p_{A}^{\alpha}-\frac{\partial L}{\partial v_{\alpha}^{A}}\right) \mathrm{d}^{m} x,
$$

which are obviously $\rho_{M}^{0}$-semibasic forms.

As an immediate consequence, when $Y_{0} \in \mathfrak{X}^{V\left(\hat{\rho}_{2}^{0}\right)}\left(\mathcal{W}_{0}\right)$, condition (8) does not depend on the derivatives of $\psi_{0}$ : is a pointwise (algebraic) condition. We can define the submanifold

$$
\mathcal{W}_{1}=\left\{(\bar{y}, \mathbf{p}) \in \mathcal{W}_{0} \mid i\left(V_{0}\right)\left(\Omega_{0}\right)_{(\bar{y}, \mathbf{p})}=0, \text { for every } V_{0} \in V\left(\hat{\rho}_{2}^{0}\right)\right\},
$$

which is called the first constraint submanifold of the Hamiltonian pre-multisymplectic system $\left(\mathcal{W}_{0}, \Omega_{0}\right)$, as every section $\psi_{0}$ solution to (8) must take values in $\mathcal{W}_{1}$. We denote by $J_{1}: \mathcal{W}_{1} \hookrightarrow \mathcal{W}_{0}$ the natural embedding.

Locally, $\mathcal{W}_{1}$ is defined in $\mathcal{W}_{0}$ by the constraints $p_{A}^{\alpha}=\partial L / \partial v_{\alpha}^{A}$. Moreover:

Proposition 2: $\mathcal{W}_{1}$ is the graph of $\widetilde{\mathcal{F} L} ;$ that is, $\mathcal{W}_{1}=\left\{(\bar{y}, \widetilde{\mathcal{F} L}(\bar{y})) \in \mathcal{W} \mid \bar{y} \in J^{1} E\right\}$.

(Proof) Consider $\bar{y} \in J^{1} E$, let $\phi: M \rightarrow E$ be a representative of $\bar{y}$, and $\mathbf{p}=\widetilde{\mathcal{F} L}(\bar{y})$. For every $U \in T_{\bar{\pi}^{1}(\bar{y})} M$, consider $V=T_{\bar{\pi}^{1}(\bar{y})} \phi(U)$ and its canonical lifting $\bar{V}=T_{\bar{\pi}^{1}(\bar{y})} j^{1} \phi(U)$. From the definition of the extended Legendre map (3) we have that $\left(T_{\bar{y}} \pi\right)^{*}(\widetilde{\mathcal{F}} \mathcal{L}(\bar{y}))=\left(\Theta_{\mathcal{L}}\right)_{\bar{y}}$, then

$$
i(\bar{V})\left[\left(T_{\bar{y}} \pi^{1}\right)^{*}(\widetilde{\mathcal{F} \mathcal{L}}(\bar{y}))\right]=i(\bar{V})\left(\Theta_{\mathcal{L}}\right)_{\bar{y}}
$$

Furthermore, as $\mathbf{p}=\widetilde{\mathcal{F} L}(\bar{y})$, we also have that

$$
\begin{aligned}
i(\bar{V})\left[\left(T_{\bar{y}} \pi^{1}\right)^{*}(\widetilde{\mathcal{F} \mathcal{L}}(\bar{y}))\right] & =i\left(T_{\bar{\pi}^{1}(\bar{y})} j^{1} \phi(U)\right)\left[\left(T_{\bar{y}} \pi^{1}\right)^{*} \mathbf{p}\right) \\
& =i\left(T_{\pi^{1}(\bar{y})}\left[\left(T_{\bar{\pi}^{1}(\bar{y})} j^{1} \phi(U)\right]\right) \mathbf{p}=i\left(T_{\bar{\pi}^{1}(\bar{y})} \phi(U)\right) \mathbf{p}=i(V) \mathbf{p} .\right.
\end{aligned}
$$

Therefore, we obtain

$$
i(U)\left(\phi^{*} \mathbf{p}\right)=i(U)\left[\left(j^{1} \phi\right) *\left(\Theta_{\mathcal{L}}\right)_{y}\right]
$$

and bearing in mind the definition of the coupling form $\mathcal{C}$, this condition becomes

$$
\left.i(U)(\mathcal{C}(\bar{y}, \mathbf{p}))=i(U)\left[\left(j^{1} \phi\right) * \Theta_{\mathcal{L}}\right)_{\bar{y}}\right]
$$

Since it holds for every $U \in T_{\bar{\pi}^{1}(\bar{y})} M$, we conclude that $\mathcal{C}(\bar{y}, \mathbf{p})=\left[\left(j^{1} \phi\right)^{*} \Theta_{\mathcal{L}}\right]_{\bar{y}}$, or equivalently, $\hat{\mathcal{C}}(\bar{y}, \mathbf{p})=\hat{L}(\bar{y}, \mathbf{p})$, where we have made use of the fact that $\Theta_{\mathcal{L}}$ is the sum of the Lagrangian density $\mathcal{L}$ and a contact form $i(\mathcal{V}) \mathcal{L}$ (vanishing by pull-back of lifted sections). This is the condition defining $\mathcal{W}_{0}$, and thus we have proved that $(\bar{y}, \widetilde{\mathcal{F}} \mathcal{L}(\bar{y})) \in \mathcal{W}_{0}$, for every $\bar{y} \in J^{1} E$; that is, graph $\widetilde{\mathcal{F} L} \subset \mathcal{W}_{0}$. Furthermore, graph $\mathcal{F} \mathcal{L}$ and $\mathcal{W}_{1}$ are defined as subsets of $\mathcal{W}_{0}$ by the same local conditions: $p_{A}^{\alpha}-\partial L / \partial v_{\alpha}^{A}=0$. So we conclude that graph $\widetilde{\mathcal{F} L}=\mathcal{W}_{1}$.

Being $\mathcal{W}_{1}$ the graph of $\mathcal{F} \mathcal{L}$, it is diffeomorphic to $J^{1} E$. Every section $\psi_{0}: M \rightarrow \mathcal{W}_{0}$ is of the form $\psi_{0}=\left(\psi_{\mathcal{L}}, \psi_{\mathcal{H}}\right)$, with $\psi_{\mathcal{L}}=\rho_{1}^{0} \circ \psi_{0}: M \rightarrow J^{1} E$, and if $\psi_{0}$ takes values in $\mathcal{W}_{1}$ then $\psi_{\mathcal{H}}=\widetilde{\mathcal{F} L}$ ${ }^{\circ} \psi_{\mathcal{L}}$. In this way, every constraint, differential equation, etc., in the unified formalism can be translated to the Lagrangian or the Hamiltonian formalisms by restriction to the first or the second factors of the product bundle. 
However, as was pointed out before, the geometric condition (8) in $\mathcal{W}_{0}$, which can be solved only for sections $\psi_{0}: M \rightarrow \mathcal{W}_{1} \subset \mathcal{W}_{0}$, is stronger than the Lagrangian condition $\psi_{\mathcal{L}}^{*} i(Z) \Omega_{\mathcal{L}}=0$, [for every $\left.Z \in \mathfrak{X}\left(J^{1} E\right)\right]$ in $J^{1} E$, which can be translated to $\mathcal{W}_{1}$ by the natural diffeomorphism between them. The reason is that $T_{\mathcal{W}_{1}} \mathcal{W}_{0}=T \mathcal{W}_{1} \oplus V_{\mathcal{W}_{1}}\left(\rho_{1}^{0}\right)$, so the additional information comes therefore from the $\rho_{1}^{0}$-vertical vectors, and it is just the holonomic condition. In fact:

Theorem 1: Let $\psi_{0}: M \rightarrow \mathcal{W}_{0}$ be a section fulfilling Eq. $(8), \psi_{0}=\left(\psi_{\mathcal{L}}, \psi_{\mathcal{H}}\right)=\left(\psi_{\mathcal{L}}, \widetilde{\mathcal{F} \mathcal{L}^{\circ}} \psi_{\mathcal{L}}\right)$, where $\psi_{\mathcal{L}}=\rho_{1}^{0} \circ \psi_{0}$. Then:

(1) $\psi_{\mathcal{L}}$ is the canonical lift of the projected section $\phi=\rho_{E}^{0} \circ \psi_{0}: M \rightarrow E$ (that is, $\psi_{\mathcal{L}}$ is a holonomic section).

(2) The section $\psi_{\mathcal{L}}=j^{1} \phi$ is a solution to the Lagrangian problem, and the section $\mu^{\circ} \psi_{\mathcal{H}}$ $=\mu \circ \widetilde{\mathcal{F} L} \circ \psi_{\mathcal{L}}=\mathcal{F} \mathcal{L} \circ j^{1} \phi$ is a solution to the Hamiltonian problem.

Conversely, for every section $\phi: M \rightarrow E$ such that $j^{1} \phi$ is solutions to the Lagrangian problem (and hence $\mathcal{F} \mathcal{L}^{\circ} j^{1} \phi$ is solution to the Hamiltonian problem) we have that the section $\psi_{0}$ $=\left(j^{1} \phi, \widetilde{\mathcal{F} L} \circ j^{1} \phi\right)$, is a solution to (8) (see Diagram 4).

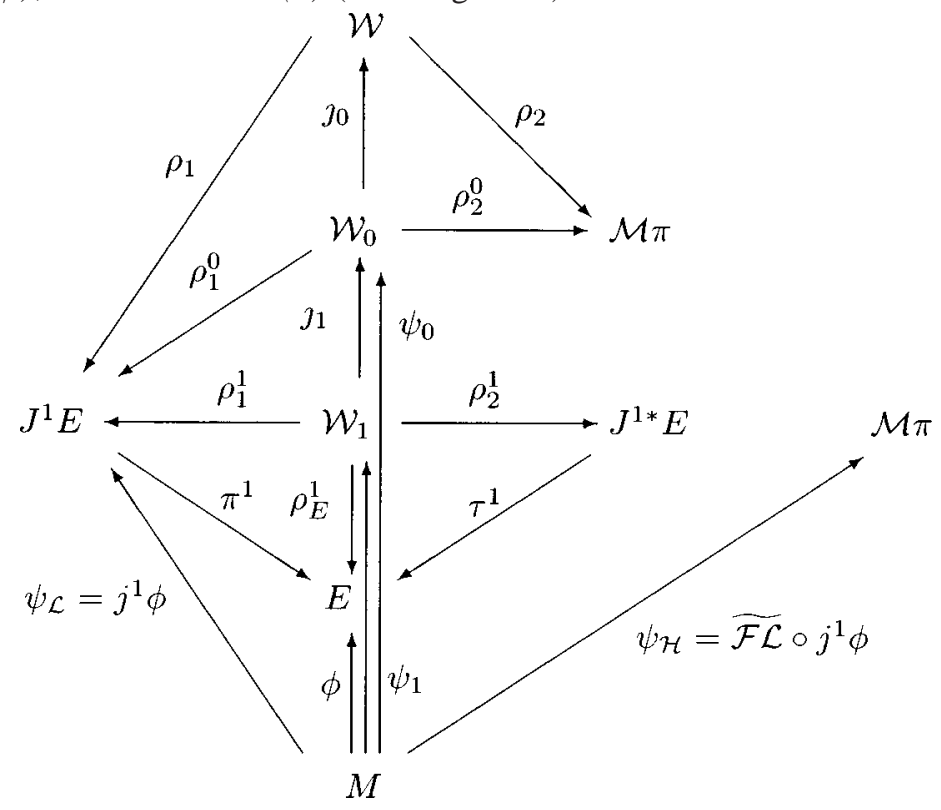

(Proof)

(1) Taking $\left\{\partial / \partial p_{A}^{\alpha}\right\}$ as a local basis for the $\rho_{1}^{0}$-vertical vector fields:

$$
i\left(\frac{\partial}{\partial p_{A}^{\alpha}}\right) \Omega_{0}=v_{\alpha}^{A} \mathrm{~d}^{m} x-\mathrm{d} y^{A} \wedge \mathrm{d}^{m-1} x_{\alpha},
$$

so that for a section $\psi_{0}$, we have

$$
0=\psi_{0}^{*}\left[i\left(\frac{\partial}{\partial p_{A}^{\alpha}}\right) \Omega_{0}\right]=\left(v_{\alpha}^{A}(x)-\frac{\partial y^{A}}{\partial x^{\alpha}}\right) \mathrm{d}^{m} x
$$

and thus the holonomy condition appears naturally within the unified formalism, and it is not necessary to impose it by hand to $\psi_{0}$. Thus, we have that $\psi_{0}=\left(x^{\alpha}, y^{A}, \partial y^{A} / \partial x^{\alpha}, \partial L / \partial v_{\alpha}^{A}\right)$, since $\psi_{0}$ takes values in $\mathcal{W}_{1}$, and hence, it is of the form $\psi_{0}=\left(j^{1} \phi, \widetilde{\mathcal{F}} \mathcal{L} \circ j^{1} \phi\right)$, for $\phi=\left(x^{\alpha}, y^{A}\right)=\rho_{E}^{0}{ }^{\circ} \psi_{0}$. 
(2) Since sections $\psi_{0}: M \rightarrow \mathcal{W}_{0}$ solution to (8) take values in $\mathcal{W}_{1}$, we can identify them with sections $\psi_{1}: M \rightarrow \mathcal{W}_{1}$. These sections $\psi_{1}$ verify, in particular, that $\psi_{1}^{*} i\left(Y_{1}\right) \Omega_{1}=0$ holds for every $Y_{1} \in \mathfrak{X}\left(\mathcal{W}_{1}\right)$. Obviously, $\psi_{0}=J_{1} \circ \psi_{1}$. Moreover, as $\mathcal{W}_{1}$ is the graph of $\mathcal{\mathcal { F }}$, denoting by $\rho_{1}^{1}=\rho_{1}^{0}$ ${ }^{\circ} J_{1}: \mathcal{W}_{1} \rightarrow J^{1} E$ the diffeomorphism which identifies $\mathcal{W}_{1}$ with $J^{1} E$, if we define $\Omega_{1}=J_{1}^{*} \Omega_{0}$, we have that $\Omega_{1}=\rho_{1}^{1 *} \Omega_{\mathcal{L}}$. In fact; as $\left(\rho_{1}^{1}\right)^{-1}(\bar{y})=(\bar{y}, \widetilde{\mathcal{F}} \mathcal{L}(\bar{y}))$, for every $\bar{y} \in J^{1} E$, then $\left(\rho_{0}^{2} J_{1}\right.$ $\left.\circ\left(\rho_{1}^{1}\right)^{-1}\right)(\bar{y})=\widetilde{\mathcal{F} L}(\bar{y}) \in \mathcal{M} \pi$, and hence,

$$
\Omega_{\mathcal{L}}=\left(\rho_{0}^{2} \circ J_{1} \circ\left(\rho_{1}^{1}\right)^{-1}\right)^{*} \Omega=\left[\left(\left(\rho_{1}^{1}\right)^{-1}\right)^{* \circ} J_{1}^{*} \circ \rho_{0}^{2 *}\right] \Omega=\left[\left(\left(\rho_{1}^{1}\right)^{-1}\right)^{*} \circ J_{1}^{*}\right] \Omega_{0}=\left(\left(\rho_{1}^{1}\right)^{-1}\right)^{*} \Omega_{1} .
$$

Now, let $X \in \mathfrak{X}\left(J^{1} E\right)$. We have

$$
\begin{aligned}
\left(j^{1} \phi\right)^{*} i(X) \Omega_{\mathcal{L}} & =\left(\rho_{1}^{0} \circ \psi_{0}\right) * i(X) \Omega_{\mathcal{L}}=\left(\rho_{1}^{0} \circ J_{1} \circ \psi_{1}\right)^{*} i(X) \Omega_{\mathcal{L}} \\
& =\left(\rho_{1}^{1} \circ \psi_{1}\right) * i(X) \Omega_{\mathcal{L}}=\psi_{1}^{*} i\left(\left(\rho_{1}^{1}\right)_{*}^{-1} X\right)\left(\rho_{1}^{1 *} \Omega_{\mathcal{L}}\right)=\psi_{1}^{*} i\left(Y_{1}\right) \Omega_{1} \\
& =\psi_{1}^{*} i\left(Y_{1}\right)\left(J_{1}^{*} \Omega_{0}\right)=\left(\psi_{1}^{*} \circ J_{1}^{*}\right) i\left(Y_{0}\right) \Omega_{0}=\psi_{0}^{*} i\left(Y_{0}\right) \Omega_{0},
\end{aligned}
$$

where $Y_{0} \in \mathfrak{X}\left(\mathcal{W}_{0}\right)$ is such that $Y_{0}=J_{1 *} Y_{1}$. But as $\psi_{0}^{*} i\left(Y_{0}\right) \Omega_{0}=0$, for every $Y_{0} \in \mathfrak{X}\left(\mathcal{W}_{0}\right)$, then we conclude that $\left(j^{1} \phi\right)^{*} i(X) \Omega_{\mathcal{L}}=0$, for every $X \in \mathfrak{X}\left(J^{1} E\right)$.

Conversely, let $j^{1} \phi: M \rightarrow J^{1} E$ such that $\left(j^{1} \phi\right)^{*} i(X) \Omega_{\mathcal{L}}=0$, for every $X \in \mathfrak{X}\left(J^{1} E\right)$, and define $\psi_{0}: M \rightarrow \mathcal{W}_{0}$ as $\psi_{0}:=\left(j^{1} \phi, \widetilde{\mathcal{F} \mathcal{L}^{\circ}} j^{1} \phi\right)$ (observe that $\psi_{0}$ takes its values in $\mathcal{W}_{1}$ ). Taking into account that, on the points of $\mathcal{W}_{1}$, every $Y_{0} \in \mathfrak{X}\left(\mathcal{W}_{0}\right)$ splits into $Y_{0}=Y_{0}^{1}+Y_{0}^{2}$, with $Y_{0}^{1} \in \mathfrak{X}\left(\mathcal{W}_{0}\right)$ tangent to $\mathcal{W}_{1}$, and $Y_{0}^{2} \in \mathfrak{X}^{V\left(\rho_{1}^{0}\right)}\left(\mathcal{W}_{0}\right)$, we have that

$$
\psi_{0}^{*} i\left(Y_{0}\right) \Omega_{0}=\psi_{0}^{*} i\left(Y_{0}^{1}\right) \Omega_{0}+\psi_{0}^{*} i\left(Y_{0}^{2}\right) \Omega_{0}=0,
$$

because for $Y_{0}^{1}$, the same reasoning as in (9) leads to

$$
\psi_{0}^{*} i\left(Y_{0}^{1}\right) \Omega_{0}=\left(j^{1} \phi\right) * i\left(X_{0}^{1}\right) \Omega_{\mathcal{L}}=0
$$

[where $\left.X_{0}^{1}=\left(\rho_{1}^{1}\right)_{*}^{-1} Y_{0}^{1}\right]$ and for $Y_{0}^{2}$, following also the same reasoning as in (9), a local calculus gives

$$
\psi_{0}^{*} i\left(Y_{0}^{2}\right) \Omega_{0}=\left(j^{1} \phi\right)^{*}\left[\left(f_{A}^{\alpha}(x)\left(v_{\alpha}^{A}-\frac{\partial y^{A}}{\partial x^{\alpha}}\right)\right) \mathrm{d}^{m} x\right]=0
$$

since $j^{1} \phi$ is a holonomic section.

The result for the sections $\mathcal{F} \mathcal{L}^{\circ} j^{1} \phi$ is a direct consequence of the equivalence theorem between the Lagrangian and Hamiltonian formalisms (see, for instance, Refs. 12 and 31).

Remark: The results in this section can also be recovered in coordinates taking an arbitrary local vector field $Y_{0}=f^{A}\left(\partial / \partial y^{A}\right)+g_{\alpha}^{A}\left(\partial / \partial v_{\alpha}^{A}\right)+h_{A}^{\alpha}\left(\partial / \partial p_{A}^{\alpha}\right) \in \mathfrak{X}\left(\mathcal{W}_{0}\right)$, then

$$
\begin{aligned}
i\left(Y_{0}\right) \Omega_{0}= & -f^{A}\left(\partial L / \partial y^{A}\right) \mathrm{d}^{m} x+f^{A} \mathrm{~d} p_{A}^{\alpha} \wedge \mathrm{d}^{m-1} x_{\alpha}+g_{\alpha}^{A}\left(p_{A}^{\alpha}-\left(\partial L / \partial v_{\alpha}^{A}\right)\right) \mathrm{d}^{m} x \\
& +h_{A}^{\alpha} v_{\alpha}^{A} \mathrm{~d}^{m} x-h_{A}^{\alpha} \mathrm{d} y^{A} \wedge \mathrm{d}^{m-1} x_{\alpha}
\end{aligned}
$$

and, for a section $\psi_{0}$ fulfilling (8), 


$$
0=\psi_{0}^{*} i\left(Y_{0}\right) \Omega_{0}=\left[f^{A}\left(\frac{\partial p_{A}^{\alpha}}{\partial x^{\alpha}}-\frac{\partial L}{\partial y^{A}}\right)+g_{\alpha}^{A}\left(p_{A}^{\alpha}-\frac{\partial L}{\partial v_{\alpha}^{A}}\right)+h_{A}^{\alpha}\left(v_{\alpha}^{A}-\frac{\partial y^{A}}{\partial x^{\alpha}}\right)\right] \mathrm{d}^{m} x
$$

reproduces the Euler-Lagrange equations, the restricted Legendre map (that is, the definition of the momenta), and the holonomy condition.

Summarizing, Eq. (8) gives different kinds of information, depending on the type of verticallity of the vector fields $Y_{0}$ involved. In particular, we have obtained equations of three different classes:

(1) Algebraic (not differential) equations, determining a subset $\mathcal{W}_{1}$ of $\mathcal{W}_{0}$, where the sections solution must take their values. These can be called primary Hamiltonian constraints, and in fact they generate, by $\hat{\rho}_{2}^{0}$ projection, the primary constraints of the Hamiltonian formalism for singular Lagrangians, i.e., the image of the Legendre transformation, $\mathcal{F} \mathcal{L}\left(J^{1} E\right) \subset J^{1 *} E$.

(2) The holonomic differential equations, forcing the sections solution $\psi_{0}$ to be lifting of $\pi$-sections. This property is similar to the one in the unified formalism of Classical Mechanics, and it reflects the fact that the geometric condition in the unified formalism is stronger than the usual one in the Lagrangian formalism.

(3) The classical Euler-Lagrange equations.

\section{Field equations for $m$-vector fields, connections, and jet fields}

The problem of finding sections solution to (8) can be formulated equivalently as follows: finding a distribution $D_{0}$ of $T\left(\mathcal{W}_{0}\right)$ such that it is integrable (that is, involutive), $m$-dimensional, $\rho_{M}^{0}$-transverse, and the integral manifolds of $D_{0}$ are the sections solution to the above equations. (Note that we do not ask them to be lifting of $\pi$-sections; that is, the holonomic condition.) This is equivalent to stating that the sections solution to this problem are the integral sections of one of the following equivalent elements:

- A class of integrable and $\rho_{M}^{0}$-transverse $m$-vector fields $\left\{X_{0}\right\} \subset \mathfrak{X}^{m}\left(\mathcal{W}_{0}\right)$ satisfying that

$$
i\left(X_{0}\right) \Omega_{0}=0, \text { for every } X_{0} \in\left\{X_{0}\right\} .
$$

- An integrable connection $\nabla_{0}$ in $\rho_{M}^{0}: \mathcal{W}_{0} \rightarrow M$ such that

$$
i\left(\nabla_{0}\right) \Omega_{0}=(m-1) \Omega_{0} .
$$

- An integrable jet field $\Psi_{0}: \mathcal{W}_{0} \rightarrow J^{1} \mathcal{W}_{0}$, such that

$$
i\left(\Psi_{0}\right) \Omega_{0}=0 .
$$

Locally decomposable and $\rho_{M}^{0}$-transverse $m$-vector fields, orientable jet fields, and orientable connections, which are solutions of these equations will be called Lagrange-Hamiltonian $m$-vector fields, jet fields, and connections for $\left(\mathcal{W}_{0}, \Omega_{0}\right)$.

Recall that, in a natural chart in $\mathcal{W}_{0}$, the local expressions of a connection form, its associated jet field, and the $m$-multivector fields of the corresponding associated class are

$$
\begin{aligned}
& \nabla_{0}=\mathrm{d} x^{\alpha} \otimes\left(\frac{\partial}{\partial x^{\alpha}}+F_{\alpha}^{A} \frac{\partial}{\partial y^{A}}+G_{\alpha \nu}^{A} \frac{\partial}{\partial v_{\nu}^{A}}+H_{\alpha A}^{\nu} \frac{\partial}{\partial p_{A}^{\nu}}\right), \\
& \Psi_{0}=\left(x^{\alpha}, y^{A}, v_{\alpha}^{A}, F_{\alpha}^{A}, G_{\alpha \eta}^{A}, H_{\alpha A}^{\nu}\right), \\
& X_{0}=f \underset{\alpha=1}{m}\left(\frac{\partial}{\partial x^{\alpha}}+F_{\alpha}^{A} \frac{\partial}{\partial y^{A}}+G_{\alpha \nu}^{A} \frac{\partial}{\partial v_{\nu}^{A}}+H_{\alpha A}^{\nu} \frac{\partial}{\partial p_{A}^{\nu}}\right),
\end{aligned}
$$


where $f \in C^{\infty}\left(J^{1} E\right)$ is an arbitrary nonvanishing function. A representative of the class $\{X\}$ can be selected by the condition $i(X)\left(\bar{\rho}_{M}^{0 *} \omega\right)=1$, which leads to $f=1$ in the above local expression.

Now, the equivalence of the unified formalism with the Lagrangian and Hamiltonian formalisms can be recovered as follows:

Theorem 2: Let $\left\{X_{0}\right\}$ be a class of integrable Lagrange-Hamiltonian m-vector fields in $\mathcal{W}_{0}$, whose elements $X_{0}: \mathcal{W}_{0} \rightarrow \Lambda^{m} T \mathcal{W}_{0}$ are solutions to (10), and let $\nabla_{0}: \mathcal{W}_{0} \rightarrow \rho_{M}^{0 *} T^{*} M \otimes_{\mathcal{W}_{0}} T \mathcal{W}_{0}$ be its associated Lagrange-Hamiltonian connection form [which is a solution to (11)], and $\Psi_{0}: \mathcal{W}_{0} \rightarrow J^{1} \mathcal{W}_{1}$ its associated Lagrange-Hamiltonian jet field [which is a solution to (12)].

(1) For every $X_{0} \in\left\{X_{0}\right\}$, the m-vector field $X_{\mathcal{L}}: J^{1} E \rightarrow \Lambda^{m} T J^{1} E$ defined by

$$
X_{\mathcal{L}^{\circ}} \rho_{1}^{0}=\Lambda^{m} T \rho_{1}^{0} \circ X_{0},
$$

is a holonomic Euler-Lagrange m-vector field for the Lagrangian system $\left(J^{1} E, \Omega_{\mathcal{L}}\right)$ (where $\Lambda^{m} T \rho_{1}^{0}: \Lambda^{m} T \mathcal{W}_{0} \rightarrow \Lambda^{m} T J^{1} E$ is the natural extension of $\left.T \rho_{1}^{0}\right)$.

Conversely, every holonomic Euler-Lagrange m-vector field for the Lagrangian system $\left(J^{1} E, \Omega_{\mathcal{L}}\right)$ can be recovered in this way from an integrable Lagrange-Hamiltonian m-vector field $X_{0} \in \mathfrak{X}_{\mathcal{W}_{1}}^{m}\left(\mathcal{W}_{0}\right)$.

(2) The Ehresmann connection form $\nabla_{\mathcal{L}}: J^{1} E \rightarrow \bar{\pi}^{1 *} T^{*} M \otimes_{J^{1} E} T J^{1} E$ defined by

$$
\nabla_{\mathcal{L}^{\circ}} \rho_{1}^{0}=\kappa_{\mathcal{W}_{0}}{ }^{\circ} \nabla_{0},
$$

is a holonomic Euler-Lagrange connection form for the Lagrangian system $\left(J^{1} E, \Omega_{\mathcal{L}}\right)$ (where $\kappa_{\mathcal{W}_{0}}$ is defined as the map making the following diagram commutative) (see Diagram 5).

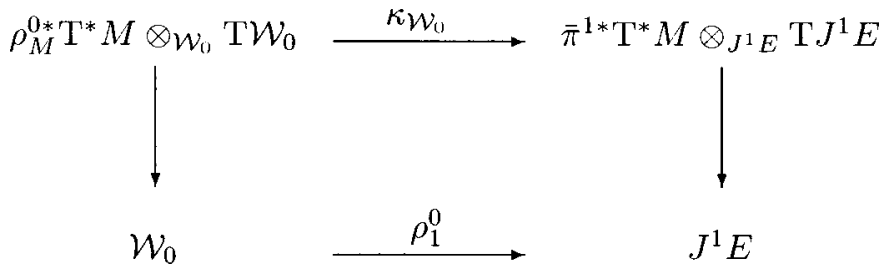

Conversely, every holonomic Euler-Lagrange connection form for the Lagrangian system $\left(J^{1} E, \Omega_{\mathcal{L}}\right)$ can be recovered in this way from an integrable Lagrange-Hamiltonian connection form $\nabla_{0}$.

(3) The jet field $\Psi_{\mathcal{L}}: J^{1} E \rightarrow J^{1} J^{1} E$ defined by

$$
\Psi_{\mathcal{L}^{\circ} \rho_{1}^{0}=j^{1} \rho_{1}^{0} \circ \Psi_{0}}
$$

is a holonomic Euler-Lagrange jet field for the Lagrangian system $\left(J^{1} E, \Omega_{\mathcal{L}}\right)$. Conversely, every holonomic Euler-Lagrange jet field for the Lagrangian system $\left(J^{1} E, \Omega_{\mathcal{L}}\right)$ can be recovered in this way from an integrable Lagrange-Hamiltonian jet field $\Psi_{0}$.

(Proof) Let $X_{0}$ be a $\rho_{M}^{0}$-transversal $m$-vector field on $\mathcal{W}_{0}$ solution to (10). As sections $\psi_{0}: M \rightarrow \mathcal{W}_{0}$ solution to the geometric equation (8) must take value in $\mathcal{W}_{1}$, then $X_{0}$ can be identified with a $m$-vector field $X_{1}: \mathcal{W}_{0} \rightarrow \Lambda^{m} T \mathcal{W}_{1}$ (i.e., $\Lambda^{m} T J_{1} \circ X_{1}=\left.X_{0}\right|_{\mathcal{W}_{1}}$ ), and hence, there exists $X_{\mathcal{L}}: J^{1} E \rightarrow \Lambda^{m} T J^{1} E$ such that $X_{1}=\Lambda^{m} T\left(\rho_{1}^{1}\right)^{-1} \circ X_{\mathcal{L}} \in \mathfrak{X}^{m}\left(\mathcal{W}_{1}\right)$. Therefore as a consequence of item (1) in theorem 1 , for every section $\psi_{0}$ solution to (8), there exists $X_{\mathcal{L}}^{0} \in \mathfrak{X}^{m}\left(j^{1} \phi(M)\right)$ such that $\Lambda^{m} T J_{\phi^{\circ}} X_{\mathcal{L}}^{0}=\left.X_{\mathcal{L}}\right|_{j}{ }^{1} \phi(M)$, where $J_{\phi}: j^{1} \phi \rightarrow E$ is the natural imbedding. So, $X_{\mathcal{L}}$ is $\bar{\pi}^{1}$-transversal and holonomic. Then, bearing in mind that $J_{1}^{*} \Omega_{0}=\rho_{1}^{1 *} \Omega_{\mathcal{L}}$, we have

$$
J_{1}^{*} i\left(X_{0}\right) \Omega_{0}=i\left(X_{1}\right)\left(J_{1}^{*} \Omega_{0}\right)=i\left(X_{1}\right)\left(\rho_{1}^{1 *} \Omega_{\mathcal{L}}\right)=\rho_{1}^{1 *} i\left(X_{\mathcal{L}}\right) \Omega_{\mathcal{L}},
$$


then $i\left(X_{0}\right) \Omega_{0}=0 \Rightarrow i\left(X_{\mathcal{L}}\right) \Omega_{\mathcal{L}}=0$.

Conversely, given an holonomic Euler-Lagrange $m$-vector field $X_{\mathcal{L}}$, from $i\left(X_{\mathcal{L}}\right) \Omega_{\mathcal{L}}=0$, and taking into account the above chain of equalities, we obtain that $i\left(X_{0}\right) \Omega_{0} \in\left[\mathfrak{X}\left(\mathcal{W}_{1}\right)\right]^{0}$ [the annihilator of $\mathfrak{X}\left(\mathcal{W}_{1}\right)$ ]. Moreover, being $X_{\mathcal{L}}$ holonomic, $X_{0}$ is holonomic, and then the extra condition $i\left(Y_{0}\right) i\left(X_{0}\right) \Omega_{0}=0$ is also fulfilled for every $Y_{0} \in \mathfrak{X}^{V\left(\rho_{1}^{0}\right)}\left(\mathcal{W}_{0}\right)$. Thus, remembering that $T_{\mathcal{W}_{1}} \mathcal{W}_{0}$ $=T \mathcal{W}_{1} \oplus \mathrm{V}_{\mathcal{W}_{1}}\left(\rho_{1}^{0}\right)$, we conclude that $i\left(X_{0}\right) \Omega_{0}=0$.

The proof for Ehresmann connections and jet fields is straightforward, taking into account that they are equivalent alternative descriptions in the Lagrangian formalism.

This statement also holds for nonintegrable classes of $m$-vector fields, connections, and jet fields in $\mathcal{W}_{0}$, but now the corresponding classes of Euler-Lagrange $m$-vector fields, connections and jet fields in $J^{1} E$ will not be holonomic (but only semi-holonomic). To prove this assertion it suffices to compute Eq. (10) in coordinates, using the local expressions (7) and (13), concluding then that, in the expressions (13), $F_{\alpha}^{A}=v_{\alpha}^{A}$, which is the local expression of the semi-holonomy condition (see, also, Ref. 9).

Finally, the Hamiltonian formalism is recovered in the usual way, by using the following:

Theorem 3: Let $\left(J^{1 *} E, \Omega_{h}\right)$ be the Hamiltonian system associated with a (hyper) regular Lagrangian system $\left(J^{1} E, \Omega_{\mathcal{L}}\right)$.

(1) (Equivalence theorem for $m$-vector fields) Let $X_{\mathcal{L}} \in \mathfrak{X}^{m}\left(J^{1} E\right)$ and $X_{\mathcal{H}} \in \mathfrak{X}^{m}\left(J^{1 *} E\right)$ be the $m$-vector fields solution to the Lagrangian and the Hamiltonian problems respectively. Then

$$
\Lambda^{m} T \mathcal{F} \mathcal{L} \circ X_{\mathcal{L}}=f X_{\mathcal{H}^{\circ}} \mathcal{F} \mathcal{L},
$$

for some $f \in C^{\infty}\left(J^{1 *} E\right)$ (we say that the classes $\left\{X_{\mathcal{L}}\right\}$ and $\left\{X_{\mathcal{H}}\right\}$ are $\mathcal{F} \mathcal{L}$-related).

(2) (Equivalence theorem for jet fields and connections) Let $\mathcal{Y}_{\mathcal{L}}$ and $\mathcal{Y}_{\mathcal{H}}$ be the jet fields solution of the Lagrangian and the Hamiltonian problems respectively. Then

$$
j^{1} \mathcal{F} \mathcal{L} \circ \mathcal{Y}_{\mathcal{L}}=\mathcal{Y}_{\mathcal{H}} \circ \mathcal{F} \mathcal{L}
$$

(we say that the jet fields $\mathcal{Y}_{\mathcal{L}}$ and $\mathcal{Y}_{\mathcal{H}}$ are $\mathcal{F} \mathcal{L}$-related). As a consequence, their associated connection forms, $\nabla_{\mathcal{L}}$ and $\nabla_{\mathcal{H}}$ respectively, are $\mathcal{F} \mathcal{L}$-related, too.

(For almost-regular systems the statement is the same, but changing $J^{1 *} E$ for $\mathcal{P}$ ).

(Proof) See Ref. 31. (The proof for the almost-regular case follows in a straight-forward way.)

As a consequence of these latter theorems, similar comments to those made at the end of Secs. II A and II B about the existence, integrability, and nonuniqueness of Euler-Lagrange and Hamilton-de Donder-Weyl $m$-vector fields, connections, and jet fields, can be applied to their associated elements in the unified formalism. In particular, for singular systems, the existence of these solutions is not assured, except perhaps on some submanifold $\mathcal{S} \hookrightarrow \mathcal{W}_{1}$, and the number of arbitrary functions which appear depends on the dimension of $\mathcal{S}$ and the rank of the Hessian matrix of $L$ (an algorithm for finding this submanifold is outlined in Ref. 9). The integrability of these solutions is not assured (even in the regular case), except perhaps on a smaller submanifold $\mathcal{I} \hookrightarrow \mathcal{S}$ such that the integral sections are contained in $\mathcal{I}$.

\section{EXAMPLE: MINIMAL SURFACES (in $\mathbb{R}^{3}$ )}

[In Ref. 9 we find another interesting example, the bosonic string (which is a singular model), described in this unified formalism.]

\section{A. Statement of the problem: Geometric elements}

The problem consists in looking for mappings $\varphi: U \subset R^{2} \rightarrow$ such that their graphs have minimal area as sets of $R^{3}$, and satisfy certain boundary conditions. 
For this model, we have that $M=\mathbb{R}^{2}, \quad E=\mathbb{R}^{2} \times \mathbb{R}$, and

$$
\begin{gathered}
J^{1} E=\pi^{*} T^{*} \mathbb{R}^{2} \otimes \mathbb{R}=\pi^{*} T^{*} M=\pi^{*} T^{*} \mathbb{R}^{2}, \\
\mathcal{M} \pi=\pi^{*}\left(T M \times{ }_{M} E\right) \quad\left(\text { affine maps from } J^{1} E \text { to } \pi^{*} \Lambda^{2} T^{*} M\right), \\
\left.J^{1 *} E=\pi^{*} T M=\pi^{*} T \mathbb{R}^{2} \text { (classes of affine maps from } J^{1} E \text { to } \pi^{*} \Lambda^{2} T^{*} M\right) .
\end{gathered}
$$

The coordinates in $J^{1} E, J^{1 *} E$ and $\mathcal{M} \pi$ are denoted $\left(x^{1}, x^{2}, y, v_{1}, v_{2}\right),\left(x^{1}, x^{2}, y, p^{1}, p^{2}\right)$, and $\left(x^{1}, x^{2}, y, p^{1}, p^{2}, p\right)$, respectively. If $\omega=\mathrm{d} x^{1} \wedge \mathrm{d} x^{2}$, the Lagrangian density is

$$
\mathcal{L}=\left[1+\left(v_{1}\right)^{2}+\left(v_{2}\right)^{2}\right]^{1 / 2} \mathrm{~d} x^{1} \wedge \mathrm{d} x^{2} \equiv L \mathrm{~d} x^{1} \wedge \mathrm{d} x^{2},
$$

and the Poincaré-Cartan forms are

$$
\begin{gathered}
\Theta_{\mathcal{L}}=\frac{v_{1}}{L} \mathrm{~d} y \wedge \mathrm{d} x^{2}-\frac{v_{2}}{L} \mathrm{~d} y \wedge \mathrm{d} x^{1}+L\left(1-\left(\frac{v_{1}}{L}\right)^{2}-\left(\frac{v_{2}}{L}\right)^{2}\right) \mathrm{d} x^{1} \wedge \mathrm{d} x^{2}, \\
\Omega_{\mathcal{L}}=-\mathrm{d}\left(\frac{v_{1}}{L}\right) \wedge \mathrm{d} y \wedge \mathrm{d} x^{2}+\mathrm{d}\left(\frac{v_{2}}{L}\right) \wedge \mathrm{d} y \wedge \mathrm{d} x^{1}-\mathrm{d}\left[L\left(1-\left(\frac{v_{1}}{L}\right)^{2}-\left(\frac{v_{2}}{L}\right)^{2}\right)\right] \wedge \mathrm{d} x^{1} \wedge \mathrm{d} x^{2} .
\end{gathered}
$$

The Legendre maps are

$$
\begin{gathered}
\mathcal{F} \mathcal{L}\left(x^{1}, x^{2}, y, v_{1}, v_{2}\right)=\left(x^{1}, x^{2}, y, \frac{v_{1}}{L}, \frac{v_{2}}{L}\right), \\
\widetilde{\mathcal{F} L}\left(x^{1}, x^{2}, y, v_{1}, v_{2}\right)=\left(x^{1}, x^{2}, y, \frac{v_{1}}{L}, \frac{v_{2}}{L}, L-\frac{\left(v_{1}\right)^{2}}{L}-\frac{\left(v_{2}\right)^{2}}{L}\right),
\end{gathered}
$$

and then $\mathcal{L}$ is hyperregular. The Hamiltonian function is

$$
H=-\left[1-\left(p^{1}\right)^{2}-\left(p^{2}\right)^{2}\right]^{1 / 2} .
$$

So the Hamilton-Cartan forms are

$$
\begin{gathered}
\Theta_{h}=p^{1} \mathrm{~d} y \wedge \mathrm{d} x^{2}-p^{2} \mathrm{~d} y \wedge \mathrm{d} x^{1}-H \mathrm{~d} x^{1} \wedge \mathrm{d} x^{2}, \\
\Omega_{h}=-\mathrm{d} p^{1} \wedge \mathrm{d} y \wedge \mathrm{d} x^{2}+\mathrm{d} p^{2} \wedge \mathrm{d} y \wedge \mathrm{d} x^{1}+\mathrm{d} H \wedge \mathrm{d} x^{1} \wedge \mathrm{d} x^{2} .
\end{gathered}
$$

\section{B. Unified formalism}

For the unified formalism we have

$$
\mathcal{W}=\pi^{*} T^{*} M \times_{E} \pi^{*}\left(T M \times_{M} E\right), \quad \mathcal{W}_{r}=\pi^{*} T^{*} M \times_{E} \pi^{*} T M=\pi^{*}\left(T^{*} M \times_{M} T M\right) .
$$

If

$$
w=\left(x^{1}, x^{2}, y, v_{1}, v_{2}, p^{1}, p^{2}, p\right) \in \mathcal{W},
$$

the coupling form is

$$
\hat{\mathcal{C}}=\left(p^{1} v_{1}+p^{2} v_{2}+p\right) \mathrm{d} x^{1} \wedge \mathrm{d} x^{2},
$$

therefore, 


$$
\mathcal{W}_{0}=\left\{\left(x^{1}, x^{2}, y, v_{1}, v_{2}, p^{1}, p^{2}, p\right) \in \mathcal{W} \mid\left[1+\left(v_{1}\right)^{2}+\left(v_{2}\right)^{2}\right]^{1 / 2}-p^{1} v_{1}-p^{2} v_{2}-p=0\right\},
$$

and we have the forms

$$
\begin{gathered}
\Theta_{0}=\left(\left[1+\left(v_{1}\right)^{2}+\left(v_{2}\right)^{2}\right]^{1 / 2}-p^{1} v_{1}-p^{2} v_{2}\right) \mathrm{d} x^{1} \wedge \mathrm{d} x^{2}-p^{2} \mathrm{~d} y \wedge \mathrm{d} x_{1}+p^{1} \mathrm{~d} y \wedge \mathrm{d} x_{2}, \\
\Omega_{0}=-\mathrm{d}\left(\left[1+\left(v_{1}\right)^{2}+\left(v_{2}\right)^{2}\right]^{1 / 2}-p^{1} v_{1}-p^{2} v_{2}\right) \wedge \mathrm{d} x^{1} \wedge \mathrm{d} x^{2}+\mathrm{d} p^{2} \wedge \mathrm{d} y \wedge \mathrm{d} x_{1}-\mathrm{d} p^{1} \wedge \mathrm{d} y \wedge \mathrm{d} x_{2} .
\end{gathered}
$$

Taking first $\hat{\rho}_{2}^{0}$-vertical vector fields $\partial / \partial v_{\alpha}$ we obtain

$$
0=i\left(\frac{\partial}{\partial v_{\alpha}}\right) \quad \Omega_{0}=\left(p^{\alpha}-\frac{v_{\alpha}}{L}\right) \mathrm{d} x^{1} \wedge \mathrm{d} x^{2},
$$

which determines the submanifold $\mathcal{W}_{1}=\operatorname{graph} \widetilde{\mathcal{F}}$ (diffeomorphic to $J^{1} E$ ), and reproduces the expression of the Legendre map. Now, taking $\rho_{1}^{0}$-vertical vector fields $\partial / \partial p^{\alpha}$, the contraction $i\left(\partial / \partial p^{\alpha}\right) \Omega_{0}$ gives, for $\alpha=1,2$, respectively,

$$
v_{1} \mathrm{~d} x^{1} \wedge \mathrm{d} x^{2}-\mathrm{d} y \wedge \mathrm{d} x^{2}, \quad v_{2} \mathrm{~d} x^{1} \wedge \mathrm{d} x^{2}+\mathrm{d} y \wedge \mathrm{d} x^{1},
$$

so that, for a section

$$
\psi_{0}=\left(x^{1}, x^{2}, y\left(x^{1}, x^{2}\right), v_{1}\left(x^{1}, x^{2}\right), v_{2}\left(x^{1}, x^{2}\right), p^{1}\left(x^{1}, x^{2}\right), p^{2}\left(x^{1}, x^{2}\right)\right),
$$

taking values in $\mathcal{W}_{1}$, we have that the condition

$$
\psi_{0}^{*}\left[i\left(\frac{\partial}{\partial p^{\alpha}}\right) \Omega_{0}\right]=0
$$

leads to

$$
\left(v_{1}-\frac{\partial y}{\partial x^{1}}\right) \mathrm{d} x^{1} \wedge \mathrm{d} x^{2}=0, \quad\left(v_{2}-\frac{\partial y}{\partial x^{2}}\right) \mathrm{d} x^{1} \wedge \mathrm{d} x^{2}=0
$$

which is the holonomy condition. Finally, taking the vector field $\partial / \partial y$ we have

$$
i\left(\frac{\partial}{\partial y}\right) \Omega_{0}=-\mathrm{d} p^{2} \wedge \mathrm{d} x^{1}+\mathrm{d} p^{1} \wedge \mathrm{d} x^{2},
$$

and, for a section $\psi_{0}$ fulfilling the former conditions, the equation

$$
0=\psi_{0}^{*}\left[i\left(\frac{\partial}{\partial y}\right) \Omega_{0}\right]
$$

leads to

$$
\begin{aligned}
0 & =\left(\frac{\partial p^{2}}{\partial x^{2}}+\frac{\partial p^{1}}{\partial x^{1}}\right) \mathrm{d} x^{1} \wedge \mathrm{d} x^{2} \\
& =\left[\frac{\partial}{\partial x^{1}}\left(\frac{v_{1}}{L}\right)+\frac{\partial}{\partial x^{2}}\left(\frac{v_{2}}{L}\right)\right] \mathrm{d} x^{1} \wedge \mathrm{d} x^{2} \\
& =\frac{1}{L^{3}}\left[\left(1+\left(\frac{\partial y}{\partial x^{1}}\right)^{2}\right) \frac{\partial^{2} y}{\partial x^{2} \partial x^{2}}+\left(1+\left(\frac{\partial y}{\partial x^{2}}\right)^{2}\right) \frac{\partial^{2} y}{\partial x^{1} \partial x^{1}}-2 \frac{\partial y}{\partial x^{1}} \frac{\partial y}{\partial x^{2}} \frac{\partial^{2} y}{\partial x^{1} \partial x^{2}}\right] \mathrm{d} x^{1} \wedge \mathrm{d} x^{2},
\end{aligned}
$$


which gives the Euler-Lagrange equation of the problem.

Now, bearing in mind (14), and the expression of the Legendre map, from the Euler-Lagrange equations we get

$$
\frac{\partial y}{\partial x^{1}}=-\frac{p^{1}}{H}, \quad \frac{\partial y}{\partial x^{2}}=-\frac{p^{2}}{H} ; \quad \frac{\partial p^{1}}{\partial x^{1}}=-\frac{\partial p^{2}}{\partial x^{2}},
$$

which are the Hamilton-De Donder-Weyl equations of the problem.

The $m$-vector fields, connections and jet fields which are the solutions to the problem in the unified formalism are

$$
\begin{gathered}
X_{0}=f\left(\frac{\partial}{\partial x^{1}}+v_{1} \frac{\partial}{\partial y}+\frac{\partial v_{1}}{\partial x^{1}} \frac{\partial}{\partial v_{1}}+\frac{\partial v_{2}}{\partial x^{1}} \frac{\partial}{\partial v_{2}}+\frac{\partial p^{1}}{\partial x^{1}} \frac{\partial}{\partial p^{1}}+\frac{\partial p^{2}}{\partial x^{1}} \frac{\partial}{\partial p^{2}}\right) \\
\wedge\left(\frac{\partial}{\partial x^{2}}+v_{2} \frac{\partial}{\partial y}+\frac{\partial v_{1}}{\partial x^{2}} \frac{\partial}{\partial v_{1}}+\frac{\partial v_{2}}{\partial x^{2}} \frac{\partial}{\partial v_{2}}+\frac{\partial p^{1}}{\partial x^{2}} \frac{\partial}{\partial p^{1}}+\frac{\partial p^{2}}{\partial x^{2}} \frac{\partial}{\partial p^{2}}\right), \\
\Psi_{0}=\left(x^{1}, x^{2}, y, p^{1}, p^{2} ; v_{1}, v_{2}, \frac{\partial v_{1}}{\partial x^{1}}, \frac{\partial v_{1}}{\partial x^{2}}, \frac{\partial v_{2}}{\partial x^{1}}, \frac{\partial v_{2}}{\partial x^{2}}, \frac{\partial p^{1}}{\partial x^{1}}, \frac{\partial p^{1}}{\partial x^{2}}, \frac{\partial p^{2}}{\partial x^{1}}, \frac{\partial p^{2}}{\partial x^{2}}\right), \\
\nabla_{0}=\mathrm{d} x^{1} \otimes\left(\frac{\partial}{\partial x^{1}}+v_{1} \frac{\partial}{\partial y}+\frac{\partial v_{1}}{\partial x^{1}} \frac{\partial}{\partial v_{1}}+\frac{\partial v_{2}}{\partial x^{1}} \frac{\partial}{\partial v_{2}}+\frac{\partial p^{1}}{\partial x^{1}} \frac{\partial}{\partial p^{1}}+\frac{\partial p^{2}}{\partial x^{1}} \frac{\partial}{\partial p^{2}}\right) \\
+\mathrm{d} x^{2} \otimes\left(\frac{\partial}{\partial x^{2}}+v_{2} \frac{\partial}{\partial y}+\frac{\partial v_{1}}{\partial x^{2}} \frac{\partial}{\partial v_{1}}+\frac{\partial v_{2}}{\partial x^{2}} \frac{\partial}{\partial v_{2}}+\frac{\partial p^{1}}{\partial x^{2}} \frac{\partial}{\partial p^{1}}+\frac{\partial p^{2}}{\partial x^{2}} \frac{\partial}{\partial p^{2}}\right)
\end{gathered}
$$

( $f$ being a nonvanishing function), where the coefficients $\partial v_{\alpha} / \partial x^{\nu}=\partial^{2} y / \partial x^{\nu} \partial x^{\alpha}$ are related by the Euler-Lagrange equations, and the coefficients $\partial p^{\alpha} / \partial x^{\nu}$ are related by the Hamilton-De DonderWeyl equations (the third one). Hence, the associated Euler-Lagrange $m$-vector fields, connections and jet fields which are the solutions to the Lagrangian problem are

$$
\begin{gathered}
X_{\mathcal{L}}=f\left(\frac{\partial}{\partial x^{1}}+v_{1} \frac{\partial}{\partial y}+\frac{\partial v_{1}}{\partial x^{1}} \frac{\partial}{\partial v_{1}}+\frac{\partial v_{2}}{\partial x^{1}} \frac{\partial}{\partial v_{2}}\right) \wedge\left(\frac{\partial}{\partial x^{2}}+v_{2} \frac{\partial}{\partial y}+\frac{\partial v_{1}}{\partial x^{2}} \frac{\partial}{\partial v_{1}}+\frac{\partial v_{2}}{\partial x^{2}} \frac{\partial}{\partial v_{2}}\right), \\
\Psi_{\mathcal{L}}=\left(x^{1}, x^{2}, y, p^{1}, p^{2} ; v_{1}, v_{2}, \frac{\partial v_{1}}{\partial x^{1}}, \frac{\partial v_{1}}{\partial x^{2}}, \frac{\partial v_{2}}{\partial x^{1}}, \frac{\partial v_{2}}{\partial x^{2}}\right), \\
\nabla_{\mathcal{L}}=\mathrm{d} x^{1} \otimes\left(\frac{\partial}{\partial x^{1}}+v_{1} \frac{\partial}{\partial y}+\frac{\partial v_{1}}{\partial x^{1}} \frac{\partial}{\partial v_{1}}+\frac{\partial v_{2}}{\partial x^{1}} \frac{\partial}{\partial v_{2}}\right)+\mathrm{d} x^{2} \otimes\left(\frac{\partial}{\partial x^{2}}+v_{2} \frac{\partial}{\partial y}+\frac{\partial v_{1}}{\partial x^{2}} \frac{\partial}{\partial v_{1}}+\frac{\partial v_{2}}{\partial x^{2}} \frac{\partial}{\partial v_{2}}\right),
\end{gathered}
$$

and the corresponding Hamilton-De Donder-Weyl $m$-vector fields, connections, and jet fields which are the solutions to the Hamiltonian problem are

$$
\begin{gathered}
X_{\mathcal{H}}=f\left(\frac{\partial}{\partial x^{1}}-\frac{p^{1}}{H} \frac{\partial}{\partial y}+\frac{\partial p^{1}}{\partial x^{1}} \frac{\partial}{\partial p^{1}}+\frac{\partial p^{2}}{\partial x^{1}} \frac{\partial}{\partial p^{2}}\right) \wedge\left(\frac{\partial}{\partial x^{2}}-\frac{p^{2}}{H} \frac{\partial}{\partial y}+\frac{\partial p^{1}}{\partial x^{2}} \frac{\partial}{\partial p^{1}}+\frac{\partial p^{2}}{\partial x^{2}} \frac{\partial}{\partial p^{2}}\right), \\
\Psi_{\mathcal{H}}=\left(x^{1}, x^{2}, y, p^{1}, p^{2} ;-\frac{p^{1}}{H},-\frac{p^{2}}{H}, \frac{\partial p^{1}}{\partial x^{1}}, \frac{\partial p^{1}}{\partial x^{2}}, \frac{\partial p^{2}}{\partial x^{1}}, \frac{\partial p^{2}}{\partial x^{2}}\right),
\end{gathered}
$$




$$
\nabla_{\mathcal{H}}=\mathrm{d} x^{1} \otimes\left(\frac{\partial}{\partial x^{1}}-\frac{p^{1}}{H} \frac{\partial}{\partial y}+\frac{\partial p^{1}}{\partial x^{1}} \frac{\partial}{\partial p^{1}}+\frac{\partial p^{2}}{\partial x^{1}} \frac{\partial}{\partial p^{2}}\right)+\mathrm{d} x^{2} \otimes\left(\frac{\partial}{\partial x^{2}}-\frac{p^{2}}{H} \frac{\partial}{\partial y}+\frac{\partial p^{1}}{\partial x^{2}} \frac{\partial}{\partial p^{1}}+\frac{\partial p^{2}}{\partial x^{2}} \frac{\partial}{\partial p^{2}}\right)
$$

\section{CONCLUSIONS AND OUTLOOK}

We have generalized the Rusk-Skinner unified formalism to first-order classical field theories. Corresponding to the Whitney sum $T Q \times{ }_{Q} T^{*} Q$ in autonomous mechanics, here we take $J^{1} E$ $\times_{E} \mathcal{M} \pi$ as standpoint, but the field equations are stated in a submanifold $\mathcal{W}_{0} \subset J^{1} E \times_{E} \mathcal{M} \pi$. As a particular case of this situation, the unified formalism for nonautonomous mechanics is recovered, the Whitney sum being now $J^{1} E \times{ }_{E} T^{*} E$, where $\pi: E \rightarrow \mathbb{R}$ is the configuration bundle. ${ }^{8,9}$ Once the suitable (pre) multisymplectic structures are introduced, the field equations can be written in several equivalent ways: using sections and vector fields (8) in $\mathcal{W}_{0}, m$-vector fields (10), connections (11), or jet fields (12).

Starting from Eq. (8), we have seen how, when different kinds of vertical vector fields in $\mathcal{W}_{0}$ are considered, this equation gives a different type of information. In particular, using $\hat{\rho}_{2}^{0}$-vertical vector fields, we can define a submanifold $\mathcal{W}_{1} \hookrightarrow \mathcal{W}_{0}$, which turns out to be the graph of the (extended) Legendre transformation (and hence diffeomorphic to $J^{1} E$ ). Furthermore, the field equations are only compatible in $\mathcal{W}_{1}$. As sections solution to the field equations take values in $\mathcal{W}_{1}$, they split in a natural way into two components, $\psi_{0}=\left(\psi_{\mathcal{L}}, \psi_{\mathcal{H}}\right)$, (with $\psi_{\mathcal{L}}: M \rightarrow J^{1} E$, and

$\psi_{\mathcal{H}}=\widetilde{\mathcal{F} L} \circ \psi_{\mathcal{L}}$ ). Then, taking $\rho_{1}^{0}$-vertical vector fields in $(8)$, we have proved that the sections solution to the field equations in the unified formalism are automatically holonomic, even in the singular case. They are so in the following sense: for every section $\psi_{0}$ solution in the unified formalism, the corresponding section $\psi_{\mathcal{L}}$ is holonomic. (As a special case, nonintegrable $m$-vector fields, connections and jet fields which are solutions to the field equations are semi-holonomic.) These solutions only exist in general in a submanifold of $\mathcal{W}_{1}$. Finally, considering (8) for a generic vector field, the Euler-Lagrange equations for $\psi_{\mathcal{L}}$, and the Hamilton-De Donder-Weyl equations for $\mu^{\circ} \psi_{\mathcal{H}}=\mathcal{F} \mathcal{L} \circ \psi_{\mathcal{L}}$ arise in a natural way. Conversely, starting from sections $\psi_{\mathcal{L}}$ $=j^{1} \phi$ and $\mathcal{F} \mathcal{L} \circ \psi_{\mathcal{L}}$ solutions to the corresponding field equations, we can recover sections $\psi_{0}$ solution to (8). Thus, we have shown the equivalence between the standard Lagrangian and Hamiltonian formalisms and the unified one. This equivalence has been also proved for $m$-vector fields, connections and jet fields.

Although the subject is not considered in this work, $\mathcal{K}$ operators (i.e., the analogous operators in field theories to the so-called evolution operator in mechanics), in their different alternative definitions ${ }^{33}$ can easily be recovered from the unified formalism, similarly to the case of classical mechanics.

In a forthcoming paper, this formalism will be applied to give a geometric framework for optimal control with partial differential equations. Although this subject has been dealt with in the context of functional analysis, to our knowledge there has been no geometric treatment of it to date.

\section{ACKNOWLEDGMENTS}

The authors acknowledge the financial support of Ministerio de Ciencia y Tecnologia, BFM2002-03493 and BFM2000-1066-C03-01. The authors thank Jeff Palmer for his assistance in preparing the English version of the manuscript.

\section{APPENDIX: $m$-VECTOR FIELDS, JET FIELDS, AND CONNECTIONS IN JET BUNDLES}

(See Refs. 17 and 27 for the proofs and other details of the following assertions.)

Let $E$ be a $n$-dimensional differentiable manifold. For $m \leqslant n$, sections of $\Lambda^{m}(T E)$ are called $m$-vector fields in $E$ (they are contravariant skew-symmetric tensors of order $m$ in $E$ ). We denote by $\mathfrak{X}^{m}(E)$ the set of $m$-vector fields in $E . Y \in \mathfrak{X}^{m}(E)$ is said to be locally decomposable if, for every $p \in E$, there exists an open neighborhood $U_{p} \subset E$ and $Y_{1}, \ldots, Y_{m} \in \mathfrak{X}\left(U_{p}\right)$ such that $Y \simeq Y_{1} \wedge \cdots \wedge Y_{m}$. Contraction of $m$-vector fields and tensor fields in $E$ is the usual one.

$U_{p}$ 
We can define the following equivalence relation: if $Y, Y^{\prime} \in \mathfrak{X}^{m}(E)$ are nonvanishing $m$-vector fields, then $Y \sim Y^{\prime}$ if there exists a nonvanishing function $f \in C^{\infty}(E)$ such that $Y^{\prime}=f Y$ (perhaps only in a connected open set $U \subseteq E$ ). Equivalence classes will be denoted by $\{Y\}$. There is a one-to-one correspondence between the set of $m$-dimensional orientable distributions $D$ in $T E$ and the set of the equivalence classes $\{Y\}$ of nonvanishing, locally decomposable $m$-vector fields in $E$. Then, there is a bijective correspondence between the set of classes of locally decomposable and $\pi$-transverse $m$-vector fields $\{Y\} \subset \mathfrak{X}^{m}(E)$, and the set of orientable jet fields $\Psi: E \rightarrow J^{1} E$; that is, the set of orientable Ehresmann connection forms $\nabla$ in $\pi: E \rightarrow M$. This correspondence is characterized by the fact that the horizontal subbundle associated with $\Psi$ (and $\nabla$ ) coincides with $\mathcal{D}(Y)$.

If $Y \in \mathfrak{X}^{m}(E)$ is nonvanishing and locally decomposable, the distribution associated with the class $\{Y\}$ is denoted $\mathcal{D}(Y)$. A nonvanishing, locally decomposable $m$-vector field $Y \in \mathfrak{X}^{m}(E)$ is said to be integrable (respectively, involutive) if its associated distribution $\mathcal{D}_{U}(Y)$ is integrable (respectively, involutive). Of course, if $Y \in \mathfrak{X}^{m}(E)$ is integrable (respectively, involutive), then so is every $m$-vector field in its equivalence class $\{Y\}$, and all of them have the same integral manifolds. Moreover, Frobenius' theorem allows us to say that a nonvanishing and locally decomposable $m$-vector field is integrable if, and only if, it is involutive. Of course, the orientable jet field $\Psi$, and the connection form $\nabla$ associated with $\{Y\}$ are integrable if, and only if, so is $Y$, for every $Y \in\{Y\}$.

Let us consider the following situation: if $\pi: E \rightarrow M$ is a fiber bundle, we are concerned with the case where the integral manifolds of integrable $m$-vector fields in $E$ are sections of $\pi$. Thus, $Y \in \mathfrak{X}^{m}(E)$ is said to be $\pi$-transverse if, at every point $y \in E,\left(i(Y)\left(\pi^{*} \beta\right)\right)_{y} \neq 0$, for every $\beta$ $\in \Omega^{m}(M)$ such that $\beta(\pi(y)) \neq 0$. Then, if $Y \in \mathfrak{X}^{m}(E)$ is integrable, it is $\pi$-transverse if its integral manifolds are local sections of $\pi$. In this case, if $\phi: U \subset M \rightarrow E$ is a local section with $\phi(x)=y$ and $\phi(U)$ is the integral manifold of $Y$ through $y$, then $T_{y}(\operatorname{Im} \phi)$ is $\mathcal{D}_{y}(Y)$. Integral sections $\phi$ of the class $\{Y\}$ can be characterized by the condition $\Lambda^{m} T \phi=f Y \circ \phi^{\circ} \sigma_{M}$, where $\sigma_{M}: \Lambda^{m} T M \rightarrow M$ is the natural projection, and $f \in C^{\infty}(E)$ is a nonvanishing function.

As a particular case, let $\{X\}: J^{1} E \rightarrow D^{m} T J^{1} E \subset\left\{\Lambda^{m} T J^{1} E\right\}$ be a class of nonvanishing, locally decomposable and $\bar{\pi}^{1}$-transverse $m$-vector fields in $J^{1} E, \Psi: J^{1} E \rightarrow J^{1} J^{1} E$ its associated jet field, and $\nabla: J^{1} E \rightarrow \bar{\pi}^{1 *} T M \otimes_{J^{1}}{ }_{E} T J^{1} E$ its associated connection form. Then, these elements are said to be holonomic if they are integrable and their integral sections $\varphi: M \rightarrow J^{1} E$ are holonomic. Furthermore, consider the $(1, m)$-tensor field in $J^{1} E$ defined by $\mathcal{J}:=i(\mathcal{V})\left(\bar{\pi}^{1} * \omega\right)$, whose local expression is $\mathcal{J}=\left(\mathrm{d} y^{A}-v_{\alpha}^{A} \mathrm{~d} x^{\alpha}\right) \wedge \mathrm{d}^{m-1} x_{\nu} \otimes \partial / \partial v_{\nu}^{A}$. A connection form $\nabla$ in $\bar{\pi}^{1}: J^{1} E \rightarrow M$ (and its associated jet field $\Psi: J^{1} E \rightarrow J^{1} J^{1} E$ ) are said to be semi-holonomic (or a second order partial differential equation), if

$$
\overbrace{\mathcal{J}\left(h^{\nabla}, \ldots, h^{\nabla}\right)}^{m}=0,
$$

where $h^{\nabla}$ denotes the horizontal projector associated with $\nabla$. If $\{X\} \subset \mathfrak{X}^{m}\left(J^{1} E\right)$ is the associated class of $\bar{\pi}^{1}$-transverse multivector fields, then this condition is equivalent to $\mathcal{J}(X)=0$, for every $X \in\{X\}$. Then the class $\{X\}$, and its associated jet field $\Psi$ and connection form $\nabla$ are holonomic if, and only if, they are integrable and semi-holonomic.

\footnotetext{
${ }^{1}$ R. Skinner and R. Rusk, J. Math. Phys. 24, 2589 (1983).

${ }^{2}$ J. F. Cariñena and C. López, Lett. Math. Phys. 14, 203 (1987).

${ }^{3}$ J. Cortés and S. Martínez, Proc. 39th IEEE Int. Conf. Decision and Control, Sydney 5216 (2000).

${ }^{4}$ J. Cortés and S. Martínez, Rep. Math. Phys. 51 (2/3), 171 (2003).

${ }^{5}$ J. Cortés, M. de León, D. Martín de Diego, and S. Martínez, SIAM J. Control Optim. 41 (5), 1389 (2002).

${ }^{6}$ M. de León, J. Cortés, D. Martín de Diego, and S. Martínez, Preprint IMAFF-CSIC (2002).

${ }^{7}$ C. López and E. Martínez, SIAM J. Control Optim. 39, 798 (2000).

${ }^{8}$ J. Cortés, S. Martínez, and F. Cantrijn, Phys. Lett. A 300, 250 (2002).

${ }^{9}$ M. de León, J. C. Marrero, and D. Martín de Diego, A New Geometrical Setting for Classical Field Theories (Banach Center, Warsawa, 2002).

${ }^{10}$ J. F. Cariñena, M. Crampin, and L. A. Ibort, Diff. Geom. Applic. 1, 345 (1991).

${ }^{11}$ A. Echeverría-Enríquez, M. C. Muñoz-Lecanda, and N. Román-Roy, Fortschr. Phys. 44, 235 (1996).

${ }^{12}$ A. Echeverría-Enríquez, M. C. Muñoz-Lecanda, and N. Román-Roy, J. Math. Phys. 41, 7402 (2000).
} 
${ }^{13}$ G. Giachetta, L. Mangiarotti, and G. Sardanashvily, New-Lagrangian and Hamiltonian Methods in Field Theory (World Scientific, Singapore, 1997).

${ }^{14}$ M. J. Gotay, in Mechanics, Analysis, and Geometry: 200 Years after Lagrange, edited by M. Francaviglia (Elsevier Science, New York, 1991), pp. 203-235.

${ }^{15}$ F. Hélein and J. Kouneiher, J. Math. Phys. 43, 2306 (2002).

${ }^{16}$ F. Hélein and J. Kouneiher, math-ph/0211046 (2002).

${ }^{17}$ M. de León, J. Marín-Solano, and J. C. Marrero, in Proceedings on New Developments in Differential Geometry, edited by L. Tamassi and J. Szenthe (Kluwer Academic, Dordrecht, 1996), pp. 291-312.

${ }^{18}$ G. Sardanashvily, Generalized Hamiltonian Formalism for Field Theory. Constraint Systems (World Scientific, Singapore, 1995).

${ }^{19}$ A. Awane, J. Math. Phys. 33, 4046 (1992).

${ }^{20}$ C. Günther, J. Diff. Geom. 25, 23 (1987).

${ }^{21}$ I. V. Kanatchikov, Rep. Math. Phys. 41, 49 (1998).

${ }^{22}$ J. Kijowski and W. M. Tulczyjew, A Symplectic Framework for Field Theories (Springer, Berlin, 1979), Vol. 170.

${ }^{23}$ M. de León, E. Merino, J. A. Oubiña, P. R. Rodrigues, and M. Salgado, J. Math. Phys. 39, 876 (1998).

${ }^{24}$ M. de León, E. Merino, and M. Salgado, J. Math. Phys. 42, 2092 (2001).

${ }^{25}$ L. K. Norris, Proc. Symp. Pure Math 54, 435 (1993).

${ }^{26}$ E. Binz, J. Sniatycki, and H. Fisher, The Geometry of Classical Fields (North-Holland, Amsterdam, 1988).

${ }^{27}$ A. Echeverría-Enríquez, M. C. Muñoz-Lecanda, and N. Román-Roy, J. Math. Phys. 39, 4578 (1998).

${ }^{28}$ P. L. García, Symp. Math. 14, 219 (1973).

${ }^{29}$ C. Paufler and H. Romer, J. Geom. Phys. 44, 52 (2002).

${ }^{30}$ D. J. Saunders, The Geometry of Jet Bundles (Cambridge, University Press, 1989), Vol. 142.

${ }^{31}$ A. Echeverría-Enríquez, M. C. Muñoz-Lecanda, and N. Román-Roy, J. Phys. A 32, 8461 (1999).

${ }^{32}$ M. J. Gotay, J. Isenberg, and J. E. Marsden, Momentum maps and classical relativistic fields, Part I: Covariant field theory, preprint (1997).

${ }^{33}$ A. Echeverría-Enríquez, J. Marín-Solano, M. C. Muñoz-Lecanda, and N. Román-Roy, Acta Appl. Math. 77, 1 (2003). 